\title{
Hybridization in Peppers (Capsicum spp.) to Improve the Volatile Composition in Fully Ripe Fruits: The Effects of Parent Combinations and Fruit Tissues
}

\author{
Estela Moreno-Peris ${ }^{1}$, Carles Cortés-Olmos ${ }^{2}$, Mónica Díez-Díaz ${ }^{3}$, M. Carmen González-Mas ${ }^{4}$, \\ Ana de Luis-Margarit ${ }^{3}\left(\mathbb{D}\right.$, Ana Fita ${ }^{2, *} \mathbb{C}$ and Adrián Rodríguez-Burruezo ${ }^{2}$ \\ 1 Escuela de Doctorado, Universidad Católica de València San Vicente Mártir. C/Guillem de Castro 65, \\ 46008 Valencia, Spain; esmope@mail.ucv.es ${ }^{2}$ Instituto de Conservación y Mejora de la Agrodiversidad \\ Valenciana (COMAV), Universitat Politècnica de València. Camino de Vera 14, 46022 Valencia, Spain; \\ carcorol@upvnet.upv.es (C.C.-O.); adrodbur@doctor.upv.es (A.R.-B.) \\ 3 Departamento de Ciencias Experimentales y Matemáticas. Facultad de Veterinaria y Ciencias Experimentales, \\ Universidad Católica de València San Vicente Mártir. C/Guillem de Castro 94, 46001 Valencia, Spain; \\ monica.diez@ucv.es (M.D.-D.); ana.deluis@ucv.es (A.d.L.-M.) \\ 4 Departamento Farmacología. Facultad de Farmacia, Universitat de València. Avda. Vicent Andrés Estellés s/n, \\ 46100 Valencia, Spain; carmen.gonzalez-mas@uv.es \\ * Correspondence: anfifer@btc.upv.es
}

Received: 16 April 2020; Accepted: 20 May 2020; Published: 22 May 2020

\begin{abstract}
Capsicum peppers (Capsicum spp.), especially C. annuum L., are one of the most important vegetables and spices in the world and their fruits are used in a range of food dishes, to provide aroma and flavor. Pungency has been largely studied, while studies on the volatile fraction are more recent and less diverse. A considerable varietal diversity among peppers has been reported in terms of the aroma quality and the qualitative and quantitative variation in the volatile fraction, particularly in fully ripe fruits, which encompass most diverse food applications and aroma profiles. Thus, a study was designed to study the inheritance of the volatile fractions in peppers and to determine if they can be improved by breeding strategies. The volatile fraction of 175 samples of ripe fruits from a diverse collection of peppers, encompassing a range of varietal types and aroma qualities, were isolated by headspace-solid-phase microextraction (HS-SPME) and analyzed by gas chromatography-mass spectrometry (GC-MS). A diverse profile of volatiles including terpenoids, esters, alkanes, and several aldehydes and alcohols, was found among the evaluated accessions. Our findings indicated that, in most cases, hybridization provided higher amounts of total volatiles and a more complex composition, particularly in the pericarp. In addition, the volatile fraction can be inherited from the parents to the offspring, as most individual volatiles in hybrids, especially major volatiles, were present in at least one of the parents, following intermediate (levels between parents) or transgressive (levels higher than the best parent) inheritance. De novo compounds (present in the hybrid, absent in the parents) were found in many samples. Comparatively, placental tissues had higher total and individual volatile levels compared with the pericarp in most parent accessions and hybrids, which must be considered by breeders if this part of the fruit is included in food formulations. By combining parent lines with complementary volatile fractions, hybridization offers a feasible method to improve the volatile composition of ripe fruits in Capsicum peppers.
\end{abstract}

Keywords: aroma; flavor; Capsicum peppers; GC-MS; HS-SPME; combining ability; inheritance models; fruit quality; hybridization 


\section{Introduction}

Originally from America, the genus Capsicum, commonly known as peppers or chiles, has been one of the most important vegetables since the 16th century [1-3]. Peppers are the fruits from five Capsicum cultivated species, namely C. annuum, C. chinense, C. frutescens, C. baccatum, and C. pubescens. More than 200 varietal types have been reported, which greatly vary in their external traits and also in their organoleptic and functional properties [4]. Among them, C. annuum is the most economically important species and is phylogenetically related to $C$. chinense and C. frutescens [3]. Peppers are now grown worldwide, with a plethora of varietal types, ecotypes, and heirlooms used fresh (both unripe or fully ripe) or dry (mainly ripe), as a vegetable and/or a spice in a range of culinary dishes, as peppers provide a myriad of flavors and aromas [5]. Also, depending on the culinary use, the pericarp or alternatively the whole fruit (i.e., a mixture of pericarp and placental tissues) is used [5].

Although peppers can be used both fully ripe or unripe, in Europe and many other areas, they are mainly consumed at the fully ripe stage. In addition, the level of bioactive/nutritional compounds increases with ripening [6,7], with aromas evolving from herbaceous/grassy notes to more diverse profiles (e.g. fruity, herbaceous, balsamic, exotic notes, etc.) and there are significant sensorial and chemical differences among varieties [8-11]. Regarding organoleptic factors, pungency is due to the presence of capsaicinoids in the fruit, which are mainly synthesized during fruit development and ripening in the placenta and septa $[12,13]$ and provide a protection system for the seeds against herbivores [14,15].

Pungency is the best-known flavor active trait in peppers and it has been studied in depth since the first studies of Scoville [6], encompassing many different approaches, including food technology, genetics and breeding, metabolomics, etc. [13,16-20]. However, studies regarding the aroma and the volatile fraction of peppers have been relatively scarce, and only in the last years, these factors have caught the attention of scientists and breeders [8-10,21,22]. Moreover, as factors related to aroma and flavor, volatiles are essential for the organoleptic quality of fresh fruits [23,24] and often promote the interest and loyalty of consumers who associate pleasant flavors and aromas with high nutritional benefits $[7,25,26]$. Thus, the aroma from any food results from its volatile fraction, which reach a level high enough to be perceived by the human nose. The whole aroma depends on the intensity, quality and combination of each odor-contributing volatile. In addition, each volatile has its specific odor detection threshold, with some that can be smelled at parts per billion, like $\beta$-damascenone (rose oil, peach, tomato, and pepino), mesifurane (strawberry and pineapple), or 3-isobutyl-2-methoxypyrazine (peppers), while others must reach much higher concentrations to be detected $[9,27,28]$. The aroma contributes to the flavor when these volatiles are detected in the retronasal area during mastication $[9,11,23]$.

Aroma and flavor preferences in peppers are very diverse, and they are partly responsible for the varietal diversity of this crop [5]. Thus, some consumers prefer hot peppers, while others prefer mild or sweet peppers. In the same way, Serrano, Numex, Jalapeno peppers, and Rocotos from the Andean region are expected to provide intense green paprika sulfurous aromas and flavors (dominated by pyrazines and other volatiles with intense grassy aroma qualities), while other markets or consumers prefer the sweet fruity aromas, even with exotic notes, from very hot Habaneros or Scotch Bonnet, or the sweet Aji Dulce from C. chinense (dominated by esters, alcohols, and short chained acids, and lack of volatiles with grassy aroma qualities) $[5,8,11]$. Other varieties, like cayenne types from $C$. annuum, C. frutescens (e.g., Tabasco varieties), or most $C$. baccatum combine green paprika and fruity aroma notes, and are highly appreciated in certain market niches $[5,21,22]$. As a result, pepper breeders have a wide range of flavor preferences to work with.

More than 300 compounds have been reported in the volatile fraction of Capsicum fruits $[4,8,9,21,29]$, although many studies have been limited to a narrow varietal diversity, belonging to specific types for food processing [23,30-33]. In addition, only a few reports have revealed an important role of the genotypic background in the diversity of the volatile fraction and aroma quality in fully ripe peppers $[9,21,22]$. Based on the less complex green peppers, inheritance was previously studied for volatile fraction expression [34]. However, the opportunities offered by hybridization to improve the 
aroma and volatile fraction of fully ripe peppers are still unknown. Thus, there is a lack of knowledge on how the volatile composition is inherited from the parents to the offspring, both quantitatively and qualitatively, as reported in some studies in Citrus, Prunus, and Solanum [27,28,35,36], among others.

Therefore, based on our previous knowledge, several sources of variation from Capsicum, encompassing different aroma qualities at the fully ripe stage were selected to assess whether breeding strategies are feasible to improve this flavor factor in peppers, despite the complexity of biosynthetic pathways of volatiles in fruits and vegetables. In the present study, the volatile fractions of a diverse set of $C$. annuum and $C$. chinense accessions, and several $C$. annuum $\times C$. annuum and some $C$. annuum $\times C$. chinense hybrids were analyzed and compared in fully ripe fruits to assess: (i) the inheritance of these flavor-active traits and (ii) the opportunity of developing new combinations of volatiles by hybridization. Furthermore, due to the relevance of both tissues in the flavor quality of foods derived from peppers, we analyzed the pericarp and placental tissues separately to determine whether the volatile composition differed among the fruit tissues.

\section{Materials and Methods}

\subsection{Plant Material}

A collection of 10 Capsicum accessions (eight $C$. annuum and two C. chinense), encompassing diverse geographical origins, varietal diversity, fruit characteristics, and aroma qualities at the fully ripe stage [3,9], were studied (Table 1). This diversity of aroma qualities was chosen in order to encompass different flavor preferences from consumers. Thus, our results were based on a comprehensive set of plant materials to inspire breeders working with different flavors and aromas. Furthermore, six $C$. annuum $\times C$. annuum and three $C$. annuum $\times C$. chinense hybrids between accessions with complementary features and aroma qualities were also evaluated.

To minimize the environmental effect on the composition of fruits, the plants were grown in a greenhouse with climate control located in the Universitat Politècnica of València (UPV), Campus de Vera, Valencia (Spain), during the spring-summer season of 2018. Ten plantlets per accession were divided into five blocks (two plants each) and transplanted to the greenhouse in February 2018, and distributed following a completely randomized factorial design. The temperature ranged from $20^{\circ} \mathrm{C}$ (night) to $28^{\circ} \mathrm{C}$ (morning), under natural light conditions. Plants were irrigated and fertilized twice a day for 5 min using a commercial $15 \mathrm{~N}-2.2 \mathrm{P}-24.9 \mathrm{~K}$ water-soluble fertilizer $\left(15 \mathrm{~N}-5 \mathrm{P}_{2} \mathrm{O}_{5}-30 \mathrm{~K}_{2} \mathrm{O}\right.$ plus micronutrients, BASF, Barcelona, Spain) at a $1 \mathrm{~g} \mathrm{~L}^{-1}$ concentration. Plants were grown according to the common practices of pepper farmers from the Mediterranean coast of Spain [37]. Phytosanitary treatments were only applied when common pests, e.g., spider mites and whiteflies, were detected.

\subsection{Preparation of Samples and Extraction of Volatiles}

Due to the genetic diversity encompassed in the experiment, the studied accessions differed considerably in fruit development and ripening and, consequently, the fruit harvest was extended for five weeks. Thus, fully ripe fruits from both parent lines and hybrids were harvested throughout July and part of August and kept at $10{ }^{\circ} \mathrm{C}$ until the analyses were performed, which was less than three days after harvesting.

A total of five composite samples per accession $(n=5)$ were prepared by mixing fruits from the two plants in eachblock, and therefore, 10 plants per accession were sampled in the experiment. Fruits from each sample were carefully washed, first with tap water and then with distilled water. Then samples were kept at $10^{\circ} \mathrm{C}$ until analysis. Just before analysis, each fruit sample was divided into two subsamples, one for each pericarp and placental tissues. The pericarp subsamples were prepared by mixing $2 \mathrm{~g}$ of fresh weight from the pericarp (free of placental tissues and septa) and finely cut into $3 \times 3 \mathrm{~mm}$ pieces and immediately transferred into a $20 \mathrm{~mL}$ sealed crimp cap headspace vial. 
Table 1. Accession name, varietal type, origin, and fruit traits of the Capsicum parent accessions and hybrids evaluated.

\begin{tabular}{|c|c|c|c|c|c|}
\hline Accession (Abbreviation) & Varietal/Pod Type & Origin & Weight (g) & Length/Width (mm) & Aroma Quality (Intensity) ${ }^{1}$ \\
\hline \multicolumn{6}{|l|}{ C. апnиuт } \\
\hline Blocky Red1 (BlockyR1) & Bell/large & Breeding line & $200-300$ & $121 / 105$ & Paprika (low) \\
\hline Blocky Red2 (BlockyR2) & Bell/intermediate & \multirow{2}{*}{$\begin{array}{l}\text { Breeding line } \\
\text { PGI Piquillo Lodosa }\end{array}$} & $150-200$ & $106 / 93$ & Paprika (low) \\
\hline Piquillo (Piquillo) & Piquillo/Ancho & & $25-50$ & $90 / 50$ & Paprika, green (intermediate) \\
\hline Chile de Arbol (Arbol) & Cayenne, small & Mexico/south USA & $5-10$ & $71 / 11$ & \multirow{4}{*}{$\begin{array}{l}\text { Paprika, green, fruity notes (high) } \\
\text { Fruity, green paprika notes (high) } \\
\text { Green, fruity notes (intermediate) } \\
\text { Green paprika, fruity citrus notes } \\
\text { (high) }\end{array}$} \\
\hline Guindilla Española (Cayenne) & Cayenne, intermediate & Valencia (Spain) & $5-10$ & $108 / 12$ & \\
\hline Pasilla Bajío (Pasilla) & Cayenne, large & Mexico/south USA & $10-25$ & $204 / 29$ & \\
\hline Chile Serrano (Serrano) & Serrano & Mexico/south USA & $5-10$ & $35 / 16$ & \\
\hline $\begin{array}{l}\text { Serrano Criollo (SerrCrio) } \\
\text { C. chinense }\end{array}$ & Serrano-Cayenne & Mexico & $<5$ & $39 / 12$ & Green paprika (interm.-high) \\
\hline PI-152225 (152225) & Cayenne & USDA & $<5$ & $41 / 12$ & \multirow{2}{*}{$\begin{array}{l}\text { Fruity, sweet, exotic (high) } \\
\text { Fruity, sweet (interm.-high) }\end{array}$} \\
\hline Ají Dulce (Ají D) & Flattened (sweet) & Cuba & $5-10$ & $20 / 31$ & \\
\hline \multicolumn{2}{|c|}{ C. anпиит $\times$ C. annuит (abbreviation) } & \multicolumn{3}{|c|}{ C. annuum $\times$ C. chinense (abbreviation) } & \\
\hline \multicolumn{2}{|c|}{ Blocky Red1×Serrano Criollo (BlockyR1×SerrCrio) } & \multicolumn{3}{|c|}{ Blocky Red1×PI-152225 (BlockyR1×152225) } & \\
\hline \multicolumn{2}{|c|}{ Blocky Red2×Serrano Criollo (BlockyR2×SerrCrio) } & \multirow{2}{*}{\multicolumn{3}{|c|}{ Blocky Red1×Ají Dulce (BlockyR1×AjiD) }} & \\
\hline \multirow{2}{*}{\multicolumn{2}{|c|}{$\begin{array}{l}\text { Piquillo×Pasilla Bajío (Piquillo } \times \text { Pasilla) } \\
\text { PiquilloxChile Serrano (Piquillo×Serrano) }\end{array}$}} & & & Blocky Red2×Ají Dulce (BlockyR2×AjiD) & \\
\hline \multirow{2}{*}{\multicolumn{5}{|c|}{$\begin{array}{l}\text { PiquilloxChile Serrano (Piquillo×Serrano) } \\
\text { Chile de Arbol } \times \text { Chile Serrano (Arbol } \times \text { Serrano) }\end{array}$}} & \\
\hline & & & & & \\
\hline \multicolumn{2}{|c|}{ Guindilla Española×Chile Serrano (Cayenne×Serrano) } & & & & \\
\hline
\end{tabular}


The time elapsed between the subsample preparation and the extraction of volatiles was $<5 \mathrm{~min}$. Placental subsamples were also prepared with $2 \mathrm{~g}$ of placenta and seeds from different fruits of the corresponding sample and followed the same preparation protocol described for the pericarp. In the case of BlockyR1×AjiD, BlockyR2×Serrano, and BlockyR2 $\times$ AjiD, it was not possible to properly separate the placenta/seeds from the pericarp to obtain useful samples due to the reduced size of the placenta in the fruits, the close contact with the pericarp, and the complex distribution of the placental tissues. Therefore, the volatile fraction was only studied in the pericarp in these hybrids.

\subsection{Extraction of the Volatile Fraction}

Headspace-solid-phase microextraction (HS-SPME) was used to isolate the volatile compounds [9,21]. A SPME holder and fiber (divinylbenzene/carboxen/polydimethylsiloxane, DVB/CAR/PDMS, 50/30 $\mu \mathrm{m}$ ) (Supelco, Bellefonte, PA, USA) were used to perform the extraction from the HS. The fiber was exposed to the headspace of the sample vials for $40 \mathrm{~min}$ at $40^{\circ} \mathrm{C}$ to allow adsorption of volatiles. The fiber was thermally desorbed at $250^{\circ} \mathrm{C}$ for $30 \mathrm{~s}$ in splitless mode in the gas chromatograph injection port, using a splitless inlet liner of $0.75 \mathrm{~mm}$ ID. Purge flow was maintained at $50 \mathrm{~mL} \mathrm{~min}^{-1}$ and purge time was $1 \mathrm{~min}$. In order to ensure that there was no cross-contamination from previous samples, the fiber was always reconditioned for $30 \mathrm{~min}$ at $250{ }^{\circ} \mathrm{C}$ in the injection port of another gas chromatograph.

\subsection{Analysis of Volatiles}

The volatile fraction was analyzed by gas chromatography-mass spectrometry (GC-MS), using a 6890 Agilent Network GC-MS system with autosampler coupled to a 5973 Inert Mass Selective detector (Agilent Technologies, Santa Clara, CA, USA). The volatile fraction was passed through a HP-5MS J\&W silica capillary column (30 m length, $0.25 \mu \mathrm{m}$ thickness film, and $0.25 \mathrm{~mm}$ i.d.), using $95 \%$ dimethylpolysiloxane and $5 \%$ phenyl as the stationary phase. The temperature of the column varied from $100^{\circ} \mathrm{C}$ to $250^{\circ} \mathrm{C}$ at a rate of $5^{\circ} \mathrm{C} \mathrm{min}-1$, maintaining $250{ }^{\circ} \mathrm{C}$ for $10 \mathrm{~min}$ at the end of each cycle $[9,21]$. A constant flow of $1 \mathrm{~mL} \mathrm{~min}^{-1}$ of helium was used as the carrier gas and the transfer line was maintained at $220^{\circ} \mathrm{C}$. The electron impact (EI) mode, at $70 \mathrm{eV}$ ionization energy at $225^{\circ} \mathrm{C}$ of source temperature, was used for the detection through the mass spectrometer. The acquisition was done in scanning mode (mass range $m / z$ 35-350 amu). The chromatograms and mass spectra were processed by means of MSD Chemstation D.02.00.275 (Agilent Technologies).

Each compound was identified by comparing its putative mass spectra with those of the NIST 2005 Mass Spectral library, compared with our previously observed GC retention times (data not shown) and also by matching with the mass spectra custom library based on the spectra of commercially available compounds. Commercially available compounds were purchased from Sigma-Aldrich Química (Madrid, Spain). When commercial standards were not available, the identification of compounds was tentatively performed based on the comparison between their mass spectra with those of the NIST 2005 Mass Spectral library. Quantification was based on the integration of the peak area of each compound, directly integrated by the TIC (total ion current chromatogram) as reported by others on Capsicum fruits $[9,21]$.

\subsection{Data Analysis}

A fixed-effects model analysis of variance (ANOVA) was performed for the GC integrated peak area data in all accessions, based on five samples per combination of accession and fruit tissue: fruit pericarp $(n=5)$ and placental tissues $(n=5)$, encompassing a total of 175 GC-MS analyses. Newman-Keuls' test (traces not considered) was used to detect differences between accession or hybrid means $(p<0.05)$ for total volatiles and individual volatiles. Statgraphics Centurion XVII software (StatGraphics Technologies, The Plains, VA, USA) was used for these statistical analyses.

Finally, in order to obtain an illustrative comparison of the volatile profiles in both parental and hybrid accessions in different fruit tissues, principal component analysis (PCA) and hierarchical cluster analysis were performed. Unit variance scaling for normalized and centered data was used for PCA, 
using Statgraphics Centurion XVII software, and hierarchical cluster analysis was performed based on distance measures from Pearson Correlations, using the ClustVis Tool [38].

\section{Results and Discussion}

Thirty-six volatile compounds were detected in the accessions and hybrids evaluated (Table 2), most of them corresponding to terpenoids, esters, and alkanes, as well as a miscellany of diverse compounds. Terpenoids (mainly non-oxygenated sesquiterpenes) were the most important group, in agreement with our previous works [9]. Several alkanes and esters, mostly related to fruity flavors $[9,21]$, were also found (Table 2). Finally, the miscellany of other compounds included a range of aroma qualities, from the strong sulfurous aroma of the green bell pepper pyrazine (3-isobutyl-2-methoxypyrazine), to the sweet/fruity/fatty notes of alcohols or the balsamic methyl salicylate [9].

Table 2. The list of volatiles identified from Capsicum accessions/hybrids with their corresponding volatile abbreviation, retention index (RI), and identification method (Id).

\begin{tabular}{|c|c|c|c|}
\hline Volatile Compound & Volatile Abbreviation & RI & $\operatorname{Id}^{1}$ \\
\hline \multicolumn{4}{|l|}{ Terpenoids } \\
\hline 3-Carene & $\mathrm{T} 1$ & 948 & $\mathrm{R}$ \\
\hline Cyclosativene & $\mathrm{T} 2$ & 1125 & $\mathrm{R}$ \\
\hline$\alpha$-Copaene & T3 & 1221 & $\mathrm{R}$ \\
\hline$\beta$-Cubebene & $\mathrm{T} 4$ & 1339 & MS \\
\hline Allo-Aromadendrene & T5 & 1386 & MS \\
\hline$\beta$-Elemene & T6 & 1398 & MS \\
\hline$\alpha$-Longipinene & T7 & 1403 & $\mathrm{R}$ \\
\hline$\alpha$-Muurolene & T8 & 1440 & MS \\
\hline$\beta$-Selinene & T9 & 1469 & MS \\
\hline$\delta$-Cadinene & T10 & 1469 & MS \\
\hline Valencene & T11 & 1474 & $\mathrm{R}$ \\
\hline$\alpha$-Himachalene & T12 & 1494 & MS \\
\hline$\beta$-Chamigrene & T13 & 1507 & $\mathrm{R}$ \\
\hline$\beta$-Himachalene & T14 & 1528 & MS \\
\hline Longipinocarvone & $\mathrm{T} 15$ & 1569 & MS \\
\hline \multicolumn{4}{|l|}{ Esters } \\
\hline Hexyl 2-methylpropanoate & E1 & 1118 & $\mathrm{R}$ \\
\hline Pentyl 4-methylpentanoate & $\mathrm{E} 2$ & 1118 & $\mathrm{R}$ \\
\hline Hexyl 2,2-dimethylpropanoate & E3 & 1197 & $\mathrm{R}$ \\
\hline Hexyl 2-methylbutanoate & E4 & 1218 & $\mathrm{R}$ \\
\hline 4-Hexen-1-yl-pentanoate & E5 & 1290 & $\mathrm{R}$ \\
\hline \multicolumn{4}{|l|}{ Alkanes } \\
\hline Tetradecane & A1 & 1413 & $\mathrm{R}$ \\
\hline 3-Methyltetradecane & A2 & 1448 & MS \\
\hline 4-Methyltetradecane & A3 & 1448 & MS \\
\hline Decylcyclopentane & A4 & 1555 & MS \\
\hline Cyclotetradecane & A5 & 1679 & MS \\
\hline Cyclopentadecane & A6 & 1799 & MS \\
\hline Nonadecane & A7 & 1910 & $\mathrm{R}$ \\
\hline 10-Methylnonadecane & A8 & 1945 & MS \\
\hline Eicosane & A9 & 2009 & $\mathrm{R}$ \\
\hline 8-Hexylpentadecane & A10 & 2045 & MS \\
\hline \multicolumn{4}{|l|}{ Miscellany/Others } \\
\hline Indole & O1 & 1174 & $\mathrm{R}$ \\
\hline 3-Isobutyl-2-methoxypyrazine & $\mathrm{O} 2$ & 1204 & $\mathrm{R}$ \\
\hline Methyl salicylate & $\mathrm{O} 3$ & 1281 & $\mathrm{R}$ \\
\hline$\alpha$-Ionone & $\mathrm{O} 4$ & 1429 & $\mathrm{R}$ \\
\hline (E)-2-hexadecen-1-ol & O5 & 1862 & MS \\
\hline (Z)-9-octadecen-1-ol & O6 & 2061 & $\mathrm{R}$ \\
\hline
\end{tabular}

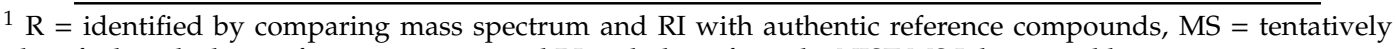
identified on the basis of mass spectrum and RI with those from the NIST MS Library and literature. 


\subsection{Volatile Fraction in the Pericarp}

\subsubsection{Parent Accessions}

The volatile composition of the fruit pericarp was very diverse among the parent accessions, both in terms of the amounts and the number of volatile compounds (Table 3). The highest amount in the total volatiles was found in 'Chile de Arbol', followed by 'Cayenne', 'Pasilla Bajio', 'Serrano', and PI-152225. These accessions also had the most diverse profiles of volatiles (Table 3). On the contrary, the rest of the accessions showed very low numbers and diversity of volatiles. These findings, particularly for the blocky/bell peppers and 'Piquillo', suggested that the total content of volatiles was highly correlated to the whole aroma intensity of these accessions (Tables 1-3).

In many accessions, the total content of volatiles was due to the accumulation of one or two volatiles (particularly terpenoids), although some other accessions showed more balanced profiles. Valencene represented about $85 \%$ of the volatiles in 'Chile de Arbol', while the rest of the compounds represented $\leq 4 \%$ of the total volatiles. Similarly, 3-carene accounted for about $95 \%$ of the total volatiles in 'Pasilla Bajio', while 3-carene and valencene in 'Cayenne' and 3-carene in PI-152225 reached almost $60 \%$ of the total volatiles (Table 3 ).

By contrast, the relative abundance of individual volatiles was distributed in a more balanced manner in 'Cayenne', PI-152225, and 'Chile Serrano'. These findings suggested that a high content in total volatiles did not necessarily correlate with a qualitatively complex volatile fraction in peppers, as previously reported [9]. Regarding individual volatiles, 'Chile de Arbol' and 'Cayenne' had complex patterns and remarkably high levels of terpenoids, followed by 'Chile Serrano', PI-152225, and 'Ají Dulce', which also had diverse terpenoid profiles but at lower levels (Table 3). However, most accessions had very low numbers of terpenoids $(\leq 5)$ (Table 3 ).

The most common and abundant terpenoids were 3-carene (penetrant terpenic sweet earthy/pine aroma description), $\alpha$-copaene (woody spicy), $\alpha$-muurolene (woody), and $\beta$-himachalene, although their relative importance depended on the accession. The terpenoid 3-carene reached relatively high levels in most accessions, particularly in 'Pasilla Bajio' and PI-152225, while valencene (citrus green/fruity aroma) was only found in four accessions, but at very high levels in 'Chile de Arbol', 'Cayenne', and 'Chile Serrano' (Table 3), suggesting that the relevance of this compound in the volatile fraction was shared by phylogenetically close small/medium-sized and thin-fleshed cayenne peppers [3]. The $\alpha$-muurolene level was much higher in 'Serrano Criollo Morelos' and C. chinense 'Aji Dulce' in comparison to other accessions, and $\alpha$-copaene was found in high amounts in 'Chile de Arbol', 'Pasilla Bajio', and 'Aji Dulce'. In contrast, despite being common in most accessions, $\alpha$-longipinene was found at very low levels. Furthermore, the contribution of the rest of terpenoids to the volatile fraction, both quantitatively and qualitatively, was low.

Esters, which usually occur at low levels in peppers and provide fruity sweet aroma notes, were only found in two $C$. annuum and the two $C$. chinense accessions. Their contribution to the volatile fraction was remarkably high in C. chinense fruits (20-25\% total volatiles), although PI-152225 showed the most complex ester profile, with pentyl 4-methylpentanoate and hexyl 2-methylbutanoate as the most abundant (Table 3). In comparison, C. annuum accessions 'Cayenne' and 'Chile Serrano' had very low levels $(<1 \%$ total volatiles) with only one ester each, hexyl 2,2-dimethylpropanoate and hexyl 2-methylpropanoate, respectively (Table 3). 
Table 3. Individual compounds with average gas chromatography (GC) peak area units $\left(\times 10^{6}\right)$ identified in the fruit pericarp of Capsicum accessions and hybrids evaluated.

\begin{tabular}{|c|c|c|c|c|c|c|c|c|c|c|c|c|c|c|c|c|c|c|c|}
\hline \multirow[b]{2}{*}{ Compound } & \multicolumn{8}{|c|}{ C. аnnuum } & \multicolumn{2}{|c|}{ C. chinense } & \multicolumn{6}{|c|}{ C. апnиит $\times$ C. annuит } & \multicolumn{3}{|c|}{ C. annuum $\times$ C. chinense } \\
\hline & BlockyR1 & BlockyR2 & Piquillo & Arbol & Cayenne & Pasilla & Serrano & SerrCrio & 152225 & $\mathrm{AjiD}$ & $\begin{array}{l}\text { BlockyR1 } \\
\text { ×SerrCrio }\end{array}$ & $\begin{array}{l}\text { BlockyR2 } \\
\times \text { ×errCrio } \\
\end{array}$ & $\begin{array}{l}\begin{array}{l}\text { Piquillo } \\
\times \text { Pasilla }\end{array} \\
\end{array}$ & $\begin{array}{l}\text { Piquillo } \\
\times \text { ×errano }\end{array}$ & $\begin{array}{c}\text { Arbol } \\
\times \text { Serrano } \\
\end{array}$ & $\begin{array}{l}\text { Cayenne } \\
\times \text { Serrano } \\
\end{array}$ & $\begin{array}{l}\text { BlockyR1 } \\
\times 152225\end{array}$ & $\begin{array}{c}\text { BlockyR1 } \\
\times A \text { AjD }\end{array}$ & $\begin{array}{c}\text { BlockyR2 } \\
\times A j i D\end{array}$ \\
\hline $\begin{array}{l}\text { Terpenoids } \\
\text { T1 }\end{array}$ & & & & & & & & & & & & & & & & & & & \\
\hline T1 & - & $2.75 \mathrm{a}^{1}$ & - & - & $36.50 \mathrm{c}$ & $188.38 \mathrm{e}$ & $2.29 \mathrm{a}$ & $6.94 a$ & 99.45d & $4.58 \mathrm{a}$ & $14.44 \mathrm{~b}$ & - & - & - & - & - & - & $1.67 \mathrm{a}$ & $20.98 \mathrm{~b}$ \\
\hline $\mathrm{T} 2$ & - & - & & - & 4.04 & 0.53 & - & - & - & - & 1.08 & - & 1.37 & & & & $\operatorname{tr}$ & - & - \\
\hline T3 & $0.96 \mathrm{a}$ & $0.44 a$ & $1.76 \mathrm{a}$ & $6.53 \mathrm{~b}$ & $098 \mathrm{~h}$ & $7.12 \mathrm{~b}$ & $1.88 \mathrm{a}$ & - & - & $8.13 \mathrm{~b}$ & 4.33ab & $0.57 \mathrm{a}$ & $2.25 \mathrm{a}$ & $3.75 \mathrm{ab}$ & $4.30 \mathrm{ab}$ & $6.18 \mathrm{~b}$ & $0.62 \mathrm{a}$ & $9.96 \mathrm{~b}$ & $112.29 \mathrm{c}$ \\
\hline T5 & - & - & - & $2.41 \mathrm{bc}$ & $0.98 \mathrm{ab}$ & - & $0.68 \mathrm{ab}$ & - & - & - & & & & $0.40 \mathrm{a}$ & $2.07 \mathrm{~b}$ & $3.34 \mathrm{c}$ & $0.01 a$ & 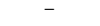 & $=$ \\
\hline $\begin{array}{l}\text { T6 } \\
\text { T7 }\end{array}$ & 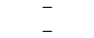 & $-\operatorname{Tr}^{2}$ & $\begin{array}{l}- \\
-\end{array}$ & $-\overline{-} 6 \mathrm{ab}$ & $\begin{array}{l}2.006 \mathrm{~b} \\
0.5 \mathrm{~b}\end{array}$ & $\begin{array}{l}- \\
-\end{array}$ & - & - & $\begin{array}{l}0.42 \mathrm{a} \\
\text { tr }\end{array}$ & - & $0.30 \mathrm{a}$ & $0.30 \mathrm{a}$ & $0.04 a$ & $0.21 \mathrm{a}$ & - & $5.40 \mathrm{c}$ & - & - & - \\
\hline T8 & - & $\begin{array}{l}1 \mathrm{r}^{2} \\
0.25 \mathrm{a}\end{array}$ & $\begin{array}{l}- \\
-\end{array}$ & $\begin{array}{c}0.20 \mathrm{ab} \\
\text { tr }\end{array}$ & $\begin{array}{l}.0 .43 \mathrm{~b} \\
2.43 \mathrm{~b}\end{array}$ & $0 . \bar{c} \mathrm{a}$ & $\begin{array}{c}0.06 a \\
\text { tr }\end{array}$ & $\begin{array}{l}0.04 \mathrm{a} \\
9.74 \mathrm{c}\end{array}$ & tr & $\begin{array}{l}0.15 \mathrm{a} \\
5.55 \mathrm{bc}\end{array}$ & $\begin{array}{l}3.00 \mathrm{c} \\
23.62 \mathrm{~d}\end{array}$ & $\begin{array}{l}0.0 \mathrm{ab} \\
849 \mathrm{c}\end{array}$ & & $\begin{array}{c}0.04 \mathrm{a} \\
\text { tr }\end{array}$ & $\begin{array}{c}0.16 a \\
\text { tr }\end{array}$ & $\begin{array}{c}0.2 \mathrm{ab} \\
\text { tr }\end{array}$ & - & $\begin{array}{l}0.6132 \mathrm{~d} \\
2132\end{array}$ & $\operatorname{tr}_{16400}$ \\
\hline T9 & $0.17 a$ & - & & & $0.41 \mathrm{a}$ & - & - & -1.74 & & -0.000 & 20.020 & -0.48 & $0.02 \mathrm{a}$ & - & - & - & $=$ & 21.520 & 164.00e \\
\hline T10 & $0.04 a$ & - & $0.01 \mathrm{a}$ & $0.12 \mathrm{a}$ & & - & - & - & $0.83 \mathrm{a}$ & $5.19 \mathrm{~b}$ & - & - & - & - & $0.11 \mathrm{a}$ & - & - & $7.63 \mathrm{~b}$ & $18.42 \mathrm{c}$ \\
\hline & & $0.06 \mathrm{a}$ & - & 205.86 & $82.62 \mathrm{c}$ & - & $68.65 \mathrm{c}$ & _- & & - & $1.66 \mathrm{a}$ & tr & $0.05 a$ & $33.19 \mathrm{~b}$ & $182.01 \mathrm{~d}$ & $285.88 \mathrm{e}$ & - & - & $34.60 \mathrm{~b}$ \\
\hline T12 & - & & - & $3.12 c$ & $1.39 \mathrm{~b}$ & - & $0.82 \mathrm{ab}$ & $0.02 \mathrm{a}$ & $1.08 \mathrm{~b}$ & 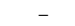 & & 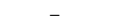 & 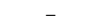 & $0.56 \mathrm{ab}$ & 270 & $3.31 \mathrm{c}$ & $0.01 \mathrm{a}$ & - & - \\
\hline & - & - & - & $\operatorname{tr}$ & & - & 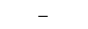 & - & $3.60 \mathrm{~b}$ & $0.10 \mathrm{a}$ & $8.32 \mathrm{c}$ & - & - & $\operatorname{tr}$ & $\operatorname{tr}$ & $\mathrm{tr}$ & $\operatorname{tr}$ & - & $118.80 \mathrm{~d}$ \\
\hline T14 & - & - & - & $7.08 \mathrm{c}$ & $5.01 \mathrm{bc}$ & - & $12.21 \mathrm{~d}$ & - & $0.55 \mathrm{a}$ & $5.28 \mathrm{bc}$ & $3.53 \mathrm{~b}$ & $0.24 a$ & - & $1.43 a b$ & $6.73 c$ & $8.13 \mathrm{c}$ & - & $8.78 \mathrm{c}$ & $53.32 \mathrm{e}$ \\
\hline T15 & - & - & - & - & - & $0.09 a$ & - & $0.03 a$ & $0.86 \mathrm{ab}$ & - & $0.19 a$ & - & $0.05 a$ & - & - & $0.81 \mathrm{ab}$ & $\begin{array}{lll}- & \\
\end{array}$ & $1.61 \mathrm{~b}$ & $8.49 \mathrm{c}$ \\
\hline $\begin{array}{c}\text { Total } \\
\text { terpenoids }\end{array}$ & $1.17 \mathrm{a}$ & 3.50a & $1.77 \mathrm{a}$ & $225.38 \mathrm{~h}$ & $135.92 \mathrm{~g}$ & $196.3 \mathrm{~h}$ & $86.59 \mathrm{f}$ & $16.77 \mathrm{c}$ & $106.79 \mathrm{fg}$ & $28.96 \mathrm{~cd}$ & $60.50 \mathrm{e}$ & $9.63 \mathrm{~b}$ & $3.78 \mathrm{a}$ & $39.58 \mathrm{~d}$ & 198.1h & $313.25 \mathrm{i}$ & $0.64 \mathrm{a}$ & $51.64 \mathrm{e}$ & $530.9 \mathrm{j}$ \\
\hline Esters & & & & & & & & & & & & & & & & & & & \\
\hline E1 & $\begin{array}{lll}- & \\
\end{array}$ & - & - & - & $\begin{array}{lll}- & \\
\end{array}$ & $\begin{array}{lll}- & \\
\end{array}$ & $0.41 \mathrm{a}$ & - & & & & $\begin{array}{lll}- & \\
\end{array}$ & $\begin{array}{lll}- & \\
\end{array}$ & $\begin{array}{lll}- & \\
\end{array}$ & - & $\begin{array}{lll}- & \\
\end{array}$ & $\begin{array}{lll}- & \\
\end{array}$ & & \\
\hline $\mathrm{E} 2$ & - & - & - & - & - & $\begin{array}{ll}- & \text { - }\end{array}$ & - & - & $16.57 \mathrm{~b}$ & $14.06 \mathrm{~b}$ & $0.37 \mathrm{a}$ & $\begin{array}{ll}- & - \\
\end{array}$ & $\begin{array}{lll}- & \\
\end{array}$ & - & - & - & $\begin{array}{ll}- & \text { r }\end{array}$ & $1.07 \mathrm{a}$ & $57.68 \mathrm{c}$ \\
\hline E3 & - & - & - & - & $1.90 \mathrm{a}$ & - & - & - & $5.84 \mathrm{~b}$ & - & $0.60 \mathrm{a}$ & - & - & $0.62 a$ & - & $28.03 \mathrm{c}$ & - & - & $39.23 \mathrm{~d}$ \\
\hline $\begin{array}{l}\text { E4 } \\
\text { E5 }\end{array}$ & - & $\begin{array}{ll}- \\
-\end{array}$ & $\begin{array}{ll}- \\
-\end{array}$ & - & - & $\begin{array}{ll}- \\
-\end{array}$ & $\begin{array}{ll}- \\
-\end{array}$ & - & $\begin{array}{l}10.15 b \\
5.49 a\end{array}$ & $\begin{array}{ll}- \\
-\end{array}$ & $\begin{array}{ll}- \\
-\end{array}$ & - & - & - & - & $1.48 \mathrm{a}$ & - & $1.25 \mathrm{a}$ & $29.00 \mathrm{c}$ \\
\hline Total esters & $\begin{array}{l}- \\
-\end{array}$ & - & $\begin{array}{ll}- \\
-\end{array}$ & - & $1.90 \mathrm{a}$ & $\begin{array}{ll}- \\
-\end{array}$ & $0.41 \mathrm{a}$ & $\overline{-}$ & $\begin{array}{l}5.49 \mathrm{ga} \\
38.05 \mathrm{c}\end{array}$ & $14.06 \mathrm{~b}$ & $0.97 \mathrm{a}$ & $\overline{-}$ & $\overline{-}$ & $0 . \overline{6} \mathrm{a}$ & $\begin{array}{ll}- \\
\end{array}$ & $29.51 \mathrm{c}$ & $\overline{-}$ & $2.32 \mathrm{a}$ & $125.91 \mathrm{~d}$ \\
\hline Alkanes & & & & & & & & & & & & & & & & & & & \\
\hline & - & - & 57 & $=1$ & & $1.09 \mathrm{a}$ & $2.22 \mathrm{~b}$ & - & 8.7 & & $1.04 a$ & $0.36 \mathrm{a}$ & $0.40 \mathrm{a}$ & & - & $6.42 \mathrm{c}$ & - & $9.24 \mathrm{~cd}$ & $11.72 \mathrm{~d}$ \\
\hline${ }^{\mathrm{A} 2}$ & $\begin{array}{ll}- \\
\end{array}$ & - & $0.07 \mathrm{a}$ & $1.51 \mathrm{ab}$ & $2.53 \mathrm{~b}$ & $0.07 \mathrm{a}$ & - & - & $1.66 \mathrm{ab}$ & $0.20 \mathrm{a}$ & $1.08 \mathrm{ab}$ & $1.23 \mathrm{ab}$ & $0.05 a$ & $1.00 \mathrm{ab}$ & $5.21 \mathrm{c}$ & - & $\begin{array}{ll}- & \text { r }\end{array}$ & - & 19.34d \\
\hline $\begin{array}{l}\mathrm{A} 3 \\
\mathrm{~A} 4\end{array}$ & $\begin{array}{l}y^{-} \\
\end{array}$ & - & - & $\begin{array}{c}- \\
0.08 \mathrm{a}\end{array}$ & - & - & $\overline{0.20 \mathrm{a}}$ & $\begin{array}{ll}- \\
-\end{array}$ & 7.05a & - & - & $0.07 \mathrm{a}$ & $\begin{array}{ll}- \\
-\end{array}$ & $4.89 \mathrm{a}$ & $0.60 \mathrm{~b}$ & - & $\begin{array}{lll}- & \\
\end{array}$ & 833ab & $\operatorname{tr}$ \\
\hline $\begin{array}{l}\text { A4 } \\
\text { A5 }\end{array}$ & $\begin{array}{ll}- \\
-\end{array}$ & $\begin{array}{ll}- \\
-\end{array}$ & $\begin{array}{ll}- \\
-\end{array}$ & $\begin{array}{l}0.08 \mathrm{a} \\
0.13 \mathrm{a}\end{array}$ & $\begin{array}{l}- \\
-\end{array}$ & $\begin{array}{ll}- \\
-\end{array}$ & $\begin{array}{l}0.20 \mathrm{a} \\
-\end{array}$ & $\begin{array}{ll}- \\
-\end{array}$ & - & $\begin{array}{ll}- \\
-\end{array}$ & $\begin{array}{c}- \\
0.22 \mathrm{a}\end{array}$ & $\begin{array}{l}0.07 \mathrm{a} \\
0.27 \mathrm{a}\end{array}$ & $\begin{array}{ll}- \\
-\end{array}$ & $\begin{array}{l}- \\
-\end{array}$ & 0.600 & $\begin{array}{ll}- \\
-\end{array}$ & $\begin{array}{ll}- \\
-\end{array}$ & $\begin{array}{l}0.335 \mathrm{ab} \\
2.35 \mathrm{~b}\end{array}$ & $15.88 \mathrm{c}$ \\
\hline A6 & - & - & - & 0.10a & $14.78 \mathrm{c}$ & $0.50 \mathrm{a}$ & - & - & $7.58 \mathrm{~b}$ & $3.06 \mathrm{ab}$ & $1.18 \mathrm{a}$ & - & $0.31 \mathrm{a}$ & 3.14ab & - & $20.35 \mathrm{~d}$ & - & $40.50 \mathrm{e}$ & $70.90 \mathrm{f}$ \\
\hline A7 & $0.08 \mathrm{a}$ & $0.03 a$ & $0.09 a$ & $0.28 \mathrm{a}$ & $2.10 \mathrm{~b}$ & it & $53.81 \mathrm{e}$ & $0.26 \mathrm{a}$ & $0.36 \mathrm{a}$ & $0.29 \mathrm{a}$ & & $0.45 a$ & $0.04 a$ & 0. tod & $1.92 \mathrm{~b}$ & . & $0.01 \mathrm{a}$ & $8.20 \mathrm{~cd}$ & $10.68 \mathrm{~d}$ \\
\hline A8 & $0.16 \mathrm{a}$ & (1) & $0.08 \mathrm{a}$ & $\begin{array}{l}8.63 c \\
0.31\end{array}$ & $42.68 \mathrm{e}$ & 1.19a & $12.36 \mathrm{~d}$ & $3.44 \mathrm{~b}$ & $14.02 \mathrm{~d}$ & & $5.37 \mathrm{bc}$ & $7.05 \mathrm{c}$ & $0.76 \mathrm{a}$ & & $11.42 \mathrm{~d}$ & $41.92 \mathrm{e}$ & $0.09 \mathrm{a}$ & $63.96 \mathrm{f}$ & $158.82 \mathrm{~g}$ \\
\hline $\begin{array}{l}\text { A9 } \\
\text { A10 }\end{array}$ & $0.03 a$ & $0.01 \mathrm{a}$ & $\begin{array}{l}0.05 a \\
0.02 a\end{array}$ & $\begin{array}{l}0.31 \mathrm{a} \\
0.44\end{array}$ & $0.55 \mathrm{a}$ & $0.03 a$ & ${ }_{10.06 \mathrm{~d}}^{10.069}$ & $0.19 \mathrm{a}$ & $0.08 \mathrm{a}$ & $0.53 a$ & $0.61 \mathrm{a}$ & $0.63 \mathrm{a}$ & $0.03 a$ & $0.28 \mathrm{a}$ & $2.18 \mathrm{~b}$ & $3.19 \mathrm{~b}$ & 0.01a & $5.70 \mathrm{c}$ & $15.39 \mathrm{e}$ \\
\hline $\begin{array}{l}\text { A10 } \\
\text { Total alkanes }\end{array}$ & $0.27 \mathrm{a}$ & $\overline{0.04 a}$ & $\begin{array}{l}0.02 \mathrm{a} \\
0.31 \mathrm{a}\end{array}$ & $\begin{array}{l}0.44 \mathrm{a} \\
11.38 \mathrm{c}\end{array}$ & $\begin{array}{l}1.05 a b \\
72.01 f\end{array}$ & $\begin{array}{l}0.03 \mathrm{a} \\
2.91 \mathrm{ab}\end{array}$ & $\begin{array}{l}4.79 \mathrm{c} \\
83.44 \mathrm{f}\end{array}$ & $\begin{array}{l}0.16 \mathrm{a} \\
4.05 \mathrm{~b}\end{array}$ & $\begin{array}{l}0.49 \mathrm{a} \\
40.03 \mathrm{e}\end{array}$ & $\begin{array}{c}0.23 \mathrm{a} \\
11.30 \mathrm{c}\end{array}$ & $\begin{array}{l}0.69 \mathrm{a} \\
10.66 \mathrm{c}\end{array}$ & $\begin{array}{l}0.39 \mathrm{a} \\
10.45 \mathrm{c}\end{array}$ & $\begin{array}{l}0.05 \mathrm{a} \\
1.64 \mathrm{ab}\end{array}$ & $\begin{array}{l}0.035 a \\
17.74 d\end{array}$ & $\begin{array}{l}1.900 \mathrm{~b} \\
23.23 \mathrm{~d}\end{array}$ & $\begin{array}{l}3.74 \mathrm{c} c \\
82.71 \mathrm{f}\end{array}$ & $\begin{array}{c}\text { tr } \\
0.11 \mathrm{a}\end{array}$ & $\begin{array}{c}4.47 \mathrm{c} \\
134.75 \mathrm{~g}\end{array}$ & $\begin{array}{l}\begin{array}{r}14.75 \mathrm{~d} \\
317.48 \mathrm{~h}\end{array} \\
\end{array}$ \\
\hline Other & & & & & & & & & & & & & & & & & & & \\
\hline $\mathrm{O} 1$ & - & $0.23 \mathrm{a}$ & - & - & $0.31 \mathrm{a}$ & $1.75 \mathrm{~b}$ & - & - & - & - & & 0.12 & - & - & & - & & - & \\
\hline $\mathrm{O}_{\mathrm{O} 2}$ & - & & $\begin{array}{lll}- & \\
- & & \end{array}$ & $0.04 a$ & $\begin{array}{lll}- & - \\
-1 & \end{array}$ & - & -0 & $0.12 \mathrm{a}$ & - & - & $1.59 \mathrm{~b}$ & & 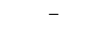 & $0.51 \mathrm{ab}$ & $0.12 \mathrm{a}$ & $\operatorname{tr}$ & $0.12 \mathrm{a}$ & - & $1.14 \mathrm{~b}$ \\
\hline 04 & $0.02 \mathrm{a}$ & $0.02 \mathrm{a}$ & - & - & - & - & $0.59 a$ & $6.28 \mathrm{c}$ & - & $1.60 \mathrm{ab}$ & 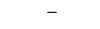 & $3.11 \mathrm{~b}$ & - & 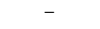 & $5.43 c$ & $\operatorname{tr}$ & $0.31 \mathrm{a}$ & $2.94 \mathrm{~b}$ & $10.63 \mathrm{~d}$ \\
\hline $\begin{array}{l}\text { O4 } \\
05\end{array}$ & - & $\begin{array}{l}- \\
-\end{array}$ & - & 3.18 & II & $\begin{array}{l}- \\
-\end{array}$ & $\begin{array}{l}- \\
-\end{array}$ & ${ }_{-}^{\operatorname{tr}}$ & $\begin{array}{l}- \\
-\end{array}$ & $\begin{array}{c}- \\
-\end{array}$ & - & $1-\bar{a}$ & $\begin{array}{l}- \\
-\end{array}$ & $\begin{array}{ll}- \\
-\end{array}$ & 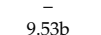 & - & - & - & - \\
\hline O6 & - & - & - & $0.20 \mathrm{da}$ & $0.20 \mathrm{a}$ & - & 1.0 & 0.06 & 1.100 & - & $0.41 \mathrm{a}$ & 0.5 & $0.02 \mathrm{a}$ & $0.11 a$ & 1.73 & 1.78 & $0.01 \mathrm{a}$ & $1.40 \mathrm{~b}$ & $6.23 \mathrm{c}$ \\
\hline Total volatiles & 1.46a & 3.79a & 2.08a & $242.34 \mathrm{e}$ & $210.37 \mathrm{e}$ & 200.96de & $172.1 \mathrm{~d}$ & $27.28 \mathrm{~b}$ & $186.02 \mathrm{~d}$ & $55.92 \mathrm{bc}$ & $74.13 \mathrm{c}$ & $25.75 \mathrm{~b}$ & 5.44a & $58.56 \mathrm{bc}$ & 237.91e & $427.25 \mathrm{f}$ & 1.19a & 193.05d & $992.29 \mathrm{~g}$ \\
\hline
\end{tabular}

${ }^{1}$ Means with different letters in the same row for each volatile indicate differences at $p<0.05$ according to Newman-Keuls test. ${ }^{2}$ tr indicates traces ( $<0.01 \times 10^{6} \mathrm{GC}$ peak area units). 
'Chile Serrano' and 'Cayenne' had the highest levels of alkanes, although the most diverse pattern was found in PI-152225. The most abundant alkanes were nonadecane and eicosane (present in all parent accessions), followed by 10-methylnonadecane and 8-hexylpentadecane (Table 3). These aliphatic hydrocarbons are thought to be related to capsaicinoid biosynthesis, and in fact, they were particularly abundant in the most pungent genotypes ('Chile Serrano' and 'Cayenne'), although their contribution to flavor and aroma was very low [9]. Methyl salicylate, a distinctive flavor-active volatile, with both green and sweet fruity notes, was usually present in cayenne types from $C$. annuum and particularly C. frutescens [9,11], while (Z)-9-octadecen-1-ol was found in four C. annuum accessions and C. chinense accessions. This fact suggests that both compounds might be intrinsic to phylogenetically close lineages of cayenne and small-fruited peppers [3,9]. Low levels of other minor compounds, like indole (floral aroma description at low levels), 3-isobutyl-2-methoxypyrazine (penetrating sulfur green pepper aroma), $\alpha$-ionone (floral aroma), and (E)-2-hexadecen-1-ol, were found in some C. annuum parental lines, but none were found in $C$. chinense. In this regard, despite that the green bell pepper, pyrazine is very common in most Capsicum species at both the unripe and fully ripe stages, and was usually absent in C. chinense ripe fruits $[8,9,11,39]$.

The fruity, sweet, and exotic aroma qualities of $C$. chinense accessions were mainly due to their relative abundance of esters, responsible for these aroma notes in fruits and vegetables, and the low content of volatiles related to green, grassy, and/or sulfur aromas, like 3-isobutyl-2-methoxypyrazine, and methyl salicylate in the case of PI-152225, as reported in other studies including this species [8,9]. The higher amounts of total volatiles and esters of PI-152225 compared to 'Aji Dulce' could be related to their higher intensity in aroma. In comparison, C. annumm peppers showed different profiles in volatiles, which is related to their varietal diversity in aromas (Table 1). The paprika aroma (at low intensity) of green and/or phenolic aroma notes in blocky peppers is due to less abundant volatile fractions, where methyl salicylate and indole (only in BlockyR2) are predominant with few terpenoids present.. The mixtures of intense paprika green aroma with fruity notes from 'Chile de Arbol' and 'Pasilla Bajio' (Table 1) is due to the combination of high levels in terpenoids (mainly valencene in 'Chile de Arbol' and 3-carene in 'Pasilla Bajio') and lack of esters in both accessions, as well as the presence of 3-isobutyl-2-methoxypyrazine (pungent sulfurous) and several alcohols in 'Chile de Arbol'. In contrast, the intense fruity green aroma with paprika notes from 'Cayenne' could be due to the comparatively higher levels of valencene than 3-carene, as well as the presence of hexyl 2,2-dimethylpropanoate. Finally, the green paprika aroma in both Serrano peppers would be due to their levels of methyl salicylate and relatively moderate levels of terpenoids, while the presence of hexyl 2-methylpropanoate and high levels of valencene in 'Chile Serrano' would contribute to its fruity citrus notes. In contrast, 3-isobutyl-2-methoxypyrazine would contribute to the earthy-sulfur notes in 'Serrano Criollo'. The differences in the aroma intensity of Serrano peppers is most likely due to differences in total volatiles (Table 3).

\subsubsection{Hybrid Accessions}

Hybrids showed more complex profiles of volatiles than their corresponding parent accessions (Table 3). For instance, the interspecific BlockyR2×AjiD hybrid showed 24 compounds, while its parents showed only 9 and 15 volatiles, respectively. In fact, only the Arbol×Serrano, Cayenne×Serrano, and BlockyR1 $\times 152225$ hybrids showed a similar or lower number of volatiles than those of the best corresponding parent (Table 3). Based on these results, both intra and interspecific hybridizations appear to be a useful breeding strategy to qualitatively increase the volatile fraction of Capsicum peppers.

The total volatiles obtained for BlockyR2 $\times$ SerrCrio, Piquillo $\times$ Pasilla, Piquillo $\times$ Serrano, Arbol $\times$ Serrano, and BlockyR1 $\times 152225$ were similar to the levels found in their corresponding parents, suggesting intermediate inheritance for this trait; however, other hybrids exceeded the range observed in the parents, suggesting transgressive inheritance: BlockyR1 $\times$ SerrCrio (3-fold the best parent), Cayenne×Serrano (2-fold), BlockyR1×AjiD (3-4-fold) and BlockyR2×AjiD (>17-fold) (Table 3).

Considering the individual compounds, most volatiles found in the hybrids were also present in at least one of the corresponding parents (Table 3), confirming the parent effect as observed in other 
vegetables, such as pepino [6]. The cases of intermediate and transgressive inheritance were variable and mainly depended on the hybrid combination. Transgressive inheritance was predominant in the volatiles of hybrids like BlockyR2×AjiD (remarkable transgressive levels of $\alpha$-muurolene, $\beta$-chamigrene, $\alpha$-copaene, cyclopentadecaene, and methyl salicylate), BlockyR1×AjiD and BlockyR1×SerrCrio, while other hybrids, like Piquillo $\times$ Pasilla, Piquillo $\times$ Serrano, and Arbol $\times$ Serrano, showed intermediate inheritance of most compounds (Table 3). Finally, some hybrids, like BlockyR $2 \times$ SerrCrio or CayennexSerrano, showed similar numbers of intermediate and transgressive inheritance. In comparison, intermediate inheritance was predominant in other studies on Citrus, Prunus, or Solanum $[27,28,35,36]$.

In addition, several cases of de novo compounds (those present in the hybrid, but absent in the parents) were found in some hybrids, particularly in BlockyR1 $\times$ SerrCrio (half of the volatiles) and to a lesser extent in BlockyR1×AjiD and BlockyR2×AjiD (Table 3), suggesting the occurrence of gene complementation [40-42]. Thus, although transgressive inheritance or genetic complementation is often observed for vigor-related traits (e.g., yield, plant development, vigor and height, fruit size, and earliness) [43], this is most likely not always the case with volatile compounds since their biosynthesis is very complex and depends on the control and regulation of a plethora of genes and their interactions $[40,42,44]$. Our findings suggested that the genotypic combinations achieved by crossing complementary parents might also result in the confluence of genetic factors silenced in both parents, contributing to the increase of the complexity of the volatile profiles in hybrids.

Overall, our results from pericarp analysis suggested that hybridization is useful to combine complementary volatile profiles, as this generally increased the total volatile content. Therefore, breeders could plan combinations of parents complementary for desirable volatiles and perform final selections based on panel tests of those hybrids with the best flavors. For instance, crosses like Cayenne $\times$ Serrano could be used to achieve new cayenne types (intermediate size) with more intense combinations of green and fruity aromas, as this hybrid had: (i) higher numbers of terpenoids than the parents and at mostly higher levels (increased woody aromas and particularly the citrus aroma from valencene that was 4-5-fold the level of the parents), (ii) remarkable increases of fruity esters, particularly hexyl 2,2-dimethyl propanoate (14-fold the values from 'Cayenne'), and (iii) the combination of traces of 3-isobutyl-2-methoxypyrazine (sulfurous green paprika aroma) and methyl salicylate (green fruity aroma), that were not present in the parents (Table 3). Breeders can also attempt to improve the aroma and flavor of modern Numex or Jalapeno varieties, crossing modern breeding lines with ancient landraces of these varietal types, which have been reported to keep more intense and attractive green paprika sulfur aromas [5]. Crosses between blocky peppers and C. chinense peppers could be used to achieve breeding materials aimed to develop fruitier exotic aromas in the blocky peppers, by introducing the synthesis of esters from C. chinense. However, these interspecific hybrids must be considered with care, due to the danger of sterility and chromosome translocations, and several combinations must be tried to select those with the most potential.

\subsection{Volatile Fraction in Placental Tissues}

\subsubsection{Parent Accessions}

Results from the placental tissues were similar to the pericarp. Thus, high total levels were not always related to diverse profiles. Again, PI-152225, 'Cayenne' and, to a lesser extent, both Serrano peppers, had the highest amounts of total volatiles and the most diverse profiles. In contrast, 'Chile de Arbol' and 'Pasilla Bajio', two accessions with considerably fewer total volatiles, also showed diverse profiles (Table 4). Finally, 'Aji Dulce' had intermediate amounts of total volatiles and low diversity, while Blocky peppers and 'Piquillo' showed the lowest contents and the least diverse profiles (Table 4). 
Table 4. Individual compounds and their average GC peak areas $\left(\times 10^{6}\right)$ identified in the placental tissues of the Capsicum accessions and hybrids evaluated.

\begin{tabular}{|c|c|c|c|c|c|c|c|c|c|c|c|c|c|c|c|c|}
\hline \multirow[b]{2}{*}{ Compound } & \multicolumn{8}{|c|}{ C. annuum } & \multicolumn{3}{|c|}{ C. chinense } & \multicolumn{3}{|c|}{ C. annuит $\times$ C. annuит } & \multicolumn{2}{|r|}{$\begin{array}{l}\text { C. annuum } \times \\
\text { C. chinense } \\
\end{array}$} \\
\hline & BlockyR1 & BlockyR2 & Piquillo & Arbol & Cayenne & Pasilla & Serrano & SerrCrio & 152225 & $\mathrm{AjiD}$ & $\begin{array}{l}\text { BlockyR1 } \\
\times \text { SerrCrio }\end{array}$ & $\begin{array}{l}\begin{array}{l}\text { Piquillo } \\
\times \text { Pasilla }\end{array} \\
\end{array}$ & $\begin{array}{l}\text { Piquillo } \\
\times \text { Serrano }\end{array}$ & $\begin{array}{c}\text { Arbol } \\
\times \text { Serrano }\end{array}$ & $\begin{array}{l}\text { Cayenne } \\
\times \text { Serrano }\end{array}$ & BlockyR1 ×152225 \\
\hline Terpenoids & & & & & & & & & & & & & & & & \\
\hline $\begin{array}{l}\mathrm{T} 1 \\
\mathrm{~T} 2\end{array}$ & $\begin{array}{l}5.56 \mathrm{r}^{2} \\
\mathrm{Tr}^{2}\end{array}$ & $\begin{array}{l}4.05 \mathrm{~b} \\
-\end{array}$ & $\begin{array}{l}8.13 \mathrm{c} \\
\mathrm{tr}\end{array}$ & $\begin{array}{l}- \\
-\end{array}$ & $51.73 \mathrm{~g}$ & $\begin{array}{l}37.50 \mathrm{f} \\
0.29 \mathrm{a}\end{array}$ & $1.42 \mathrm{a}$ & 15.84de & 15.20de & $11.98 \mathrm{~d}$ & $\begin{array}{l}17.07 \mathrm{e} \\
-\end{array}$ & $-\bar{c}$ & $2.03 a$ & - & $2.94 \mathrm{a}$ & - \\
\hline T3 & $1.35 \mathrm{ab}$ & $0.28 \mathrm{a}$ & $0.47 a$ & $2.67 \mathrm{~b}$ & - & $\begin{array}{l}0.29 \mathrm{a} \\
2.78 \mathrm{~b}\end{array}$ & $7.89 \mathrm{c}$ & $5.95 \mathrm{c}$ & $\overline{-}$ & $68.88 \mathrm{f}$ & $14.38 \mathrm{~d}$ & $\begin{array}{l}3.11 \mathrm{a} \\
3.18 \mathrm{~b}\end{array}$ & $8.50 \mathrm{c}$ & $12.55 \mathrm{~d}$ & $25.28 \mathrm{e}$ & $0 . \overline{4} 4 \mathrm{a}$ \\
\hline $\mathrm{T} 4$ & - & - & - & $\operatorname{tr}$ & & - & & - & - & - & - & - & - & 0.68 & & - \\
\hline T5 & - & - & - & $1.29 \mathrm{a}$ & $2.79 \mathrm{ab}$ & - & $3.94 \mathrm{~b}$ & - & - & - & - & - & $3.53 \mathrm{~b}$ & $8.63 \mathrm{c}$ & $15.87 \mathrm{~d}$ & - \\
\hline T6 & - & - & - & - & $27.14 \mathrm{e}$ & $0.87 \mathrm{a}$ & (2) & - & $3.21 \mathrm{~b}$ & - & $8.82 \mathrm{~d}$ & $1.95 \mathrm{~b}$ & $4.26 \mathrm{c}$ & & $21.39 \mathrm{e}$ & $0.38 a$ \\
\hline T7 & $0.01 \mathrm{a}$ & - & - & $0.11 \mathrm{a}$ & $2.94 \mathrm{c}$ & $0.04 a$ & $0.22 \mathrm{a}$ & $0.83 \mathrm{~b}$ & & - & $2.02 \mathrm{c}$ & $\operatorname{tr}$ & $0.25 a$ & $0.42 \mathrm{ab}$ & $0.78 \mathrm{~b}$ & $0.05 a$ \\
\hline $\begin{array}{l}\text { T8 } \\
\text { T9 }\end{array}$ & - & $0.73 a$ & - & $\operatorname{tr}$ & $35.23 \mathrm{~d}$ & tr & $\operatorname{tr}$ & $301.91 \mathrm{e}$ & $24.86 \mathrm{c}$ & $37.82 \mathrm{~d}$ & - & $4.93 \mathrm{~b}$ & - & - & - & $22.25 \mathrm{c}$ \\
\hline $\begin{array}{l}\text { T9 } \\
\text { T10 }\end{array}$ & $0.01 \mathrm{a}$ & - & $0.05 a$ & $\begin{array}{l}0.31 \mathrm{~b} \\
0.04 \mathrm{a}\end{array}$ & $\stackrel{-}{0.27 \mathrm{ab}}$ & - & - & - & $\begin{array}{c}- \\
4.24 \mathrm{c}\end{array}$ & 39268 & - & $0.05 a$ & - & -- & $068 \mathrm{~b}$ & - \\
\hline T11 & $0.17 \mathrm{a}$ & $0 . \overline{14 a}$ & - & $\begin{array}{l}0.04 \mathrm{a} \\
110.69 \mathrm{~b}\end{array}$ & $\begin{array}{l}0.2 \mathrm{LaO} \\
253.97 \mathrm{~cd}\end{array}$ & $0.55 a$ & $404.62 \mathrm{e}$ & - & $\begin{array}{l}4.294 \mathrm{c} c \\
225 \mathrm{c}\end{array}$ & $39.26 \mathrm{~d}$ & $1297.48 \mathrm{~g}$ & $0.08 \mathrm{a}$ & $207.91 \mathrm{~d}$ & $\begin{array}{l}0.30 \mathrm{ab} \\
730.40 \mathrm{f}\end{array}$ & $\begin{array}{c}0.68 \mathrm{~b} \\
1311.56 \mathrm{~g}\end{array}$ & - \\
\hline & & & - & $1.93 \mathrm{ab}$ & $3.69 \mathrm{~b}$ & - & $3.43 \mathrm{~b}$ & $1.20 \mathrm{ab}$ & 9.48de & - & 127.408 & . & $\begin{array}{l}2.79 \mathrm{c} \\
4.49 \mathrm{c}\end{array}$ & $8.72 \mathrm{~d}$ & $11.42 \mathrm{e}$ & $0 .-1 \mathrm{a}$ \\
\hline Т13 & _- & - & - & tr & & - & & tr & & $0.89 \mathrm{a}$ & $422.36 \mathrm{~b}$ & $\operatorname{tr}$ & & tr & & $0.11 \mathrm{~d}$ \\
\hline T14 & & - & - & $4.57 \mathrm{ab}$ & 29.31e & $\operatorname{tr}$ & $8.27 \mathrm{bc}$ & $11.20 \mathrm{c}$ & $56.55 \mathrm{f}$ & - & $174.29 \mathrm{~g}$ & $1.65 \mathrm{a}$ & $10.40 \mathrm{c}$ & $22.26 \mathrm{~d}$ & $28.97 \mathrm{e}$ & - \\
\hline T15 & $0.06 \mathrm{a}$ & $0.02 \mathrm{a}$ & $0.07 \mathrm{a}$ & - & 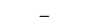 & $0.46 \mathrm{~b}$ & 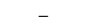 & 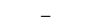 & $5.87 \mathrm{~d}$ & 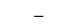 & $2.59 \mathrm{c}$ & $0.21 \mathrm{ab}$ & 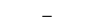 & 2.2. & $3.92 \mathrm{c}$ & $0.05 \mathrm{a}$ \\
\hline Total terpenoids & $7.16 \mathrm{a}$ & $5.22 \mathrm{a}$ & $8.72 \mathrm{ab}$ & $121.61 \mathrm{f}$ & $407.07 \mathrm{i}$ & $42.49 \mathrm{e}$ & $429.79 \mathrm{i}$ & $336.93 \mathrm{~h}$ & $348.66 \mathrm{~h}$ & $158.83 \mathrm{~g}$ & $1516.65 \mathrm{k}$ & $12.22 \mathrm{~b}$ & $331.43 \mathrm{~h}$ & $783.99 \mathrm{j}$ & $1422.81 \mathrm{k}$ & $23.28 \mathrm{c}$ \\
\hline Esters & & & & & & & & & & & & & & & & \\
\hline E1 & - & - & - & - & $\begin{array}{l}7.74 \mathrm{~b} \\
430 \mathrm{a}\end{array}$ & - & - & $37.03 d$ & $5.27 \mathrm{~b}$ & - & - & - & - & $1.22 \mathrm{a}$ & $19.60 \mathrm{c}$ & 3.83ab \\
\hline $\begin{array}{l}\text { E2 } \\
\text { E3 }\end{array}$ & $\begin{array}{l}- \\
-\end{array}$ & $\begin{array}{l}- \\
-\end{array}$ & - & 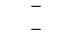 & $\begin{array}{l}43.109 \mathrm{~b} \\
11749 \mathrm{~g}\end{array}$ & - & $-\overline{-} .46 \mathrm{~b}$ & -- & $\begin{array}{l}149.4 \mathrm{c} c \\
38.17 \mathrm{e}\end{array}$ & $-\overline{52.22 \mathrm{f}}$ & $\begin{array}{l}5.5 \mathrm{a} \\
8.14 \mathrm{c}\end{array}$ & - & -- & $8.85 \mathrm{a}$ & $14280 \mathrm{~h}$ & $8 .-$ \\
\hline $\begin{array}{l}\mathrm{E} 3 \\
\mathrm{E} 4\end{array}$ & - & & - & - & & $0.52 \mathrm{a}$ & $2.46 \mathrm{~b}$ & & $\begin{array}{l}50.1 / \mathrm{e} \\
99.01 \mathrm{c}\end{array}$ & -52.221 & $\begin{array}{l}\begin{array}{l}8.14 \mathrm{c} \\
0.91 \mathrm{a}\end{array}\end{array}$ & - & $0.98 \mathrm{~d}$ & 10.150 & $\begin{array}{l}142.80 \mathrm{n} \\
10.66 \mathrm{~b}\end{array}$ & $\begin{array}{l}0.02 \mathrm{c} \\
0.97 \mathrm{a}\end{array}$ \\
\hline E5 & - & - & - & - & $2.54 \mathrm{c}$ & $0.03 a$ & - & 22.91d & $49.46 \mathrm{e}$ & - & - & - & - & - & -0 & $0.55 \mathrm{~b}$ \\
\hline Total esters & - & - & - & - & $170.86 \mathrm{f}$ & $0.55 a$ & $2.46 \mathrm{~b}$ & $204.13 \mathrm{f}$ & $341.38 \mathrm{~g}$ & $52.22 \mathrm{e}$ & $14.8 \mathrm{c}$ & - & $0.98 \mathrm{a}$ & $25.22 \mathrm{~d}$ & $173.06 \mathrm{f}$ & $13.87 \mathrm{c}$ \\
\hline Alkanes & & & & & & & & & & & & & & & & \\
\hline & $0.76 \mathrm{a}$ & $0.40 \mathrm{a}$ & $0.45 \mathrm{a}$ & $0.83 \mathrm{a}$ & 42.74 & $3.37 \mathrm{~b}$ & $16.91 \mathrm{~d}$ & $3.11 \mathrm{~b}$ & $31.44 \mathrm{e}$ & - & $3.19 \mathrm{~b}$ & $0.97 \mathrm{a}$ & $6.48 \mathrm{c}$ & $5.67 \mathrm{c}$ & $43.58 \mathrm{f}$ & $0.38 \mathrm{a}$ \\
\hline A2 & - & - & $0.09 a$ & $4.55 \mathrm{~d}$ & $19.27 \mathrm{f}$ & $0.86 \mathrm{a}$ & - & - & $9.64 \mathrm{e}$ & $2.33 \mathrm{c}$ & $45.81 \mathrm{~g}$ & - & - & $24.75 \mathrm{f}$ & - & $1.25 \mathrm{~b}$ \\
\hline A3 & - & - & - & - & - & - & - & - & - & - & - & - & - & & 248.29a & - \\
\hline A4 & - & - & - & $0.69 \mathrm{~b}$ & $1.31 \mathrm{c}$ & - & $2.01 \mathrm{~d}$ & $0.86 \mathrm{~b}$ & - & - & - & $0.11 \mathrm{a}$ & $1.13 \mathrm{bc}$ & $3.19 \mathrm{e}$ & $2.40 \mathrm{~d}$ & $\operatorname{tr}$ \\
\hline A5 & - & - & - & - & $6.49 \mathrm{~d}$ & $0.13 \mathrm{a}$ & $\begin{array}{l}1.03 \mathrm{~b} \\
6553 \mathrm{O}\end{array}$ & - & - & - & $3.90 \mathrm{c}$ & $0.11 \mathrm{a}$ & $0.78 \mathrm{ab}$ & $1.76 \mathrm{~b}$ & 0625 & ד \\
\hline $\begin{array}{l}\mathrm{A} 6 \\
\mathrm{A7}\end{array}$ & $0.16 \mathrm{a}$ & - & - & $\begin{array}{l}4.30 \mathrm{~b} \\
0.94\end{array}$ & & $3.94 \mathrm{~b}$ & $\begin{array}{l}64.53 \mathrm{e} \\
756 \mathrm{~g}\end{array}$ & - & $103.96 \mathrm{f}$ & - & $33.51 \mathrm{c}$ & $4.46 \mathrm{~b}$ & 756 & $42.59 \mathrm{~d}$ & $96.35 \mathrm{f}$ & $3.22 \mathrm{~b}$ \\
\hline $\begin{array}{l}\text { A7 } \\
\text { A8 }\end{array}$ & $\begin{array}{l}0.12 \mathrm{a} \\
2.35 \mathrm{~b}\end{array}$ & $\begin{array}{l}0.04 a \\
0.09 a\end{array}$ & $\begin{array}{l}0.08 a \\
1.41 a\end{array}$ & $\begin{array}{l}0.94 \mathrm{a} \\
8.25 \mathrm{c}\end{array}$ & $\begin{array}{l}15.91 \mathrm{e} \\
25574 \mathrm{~g}\end{array}$ & $\begin{array}{l}0.51 \mathrm{la} \\
13.98 \mathrm{~d}\end{array}$ & $\begin{array}{l}77.566 \mathrm{~g} \\
83.55 \mathrm{f}\end{array}$ & $\begin{array}{l}8.04 \mathrm{cc} \\
85.63 \mathrm{f}\end{array}$ & $\begin{array}{l}3.34 \mathrm{~b} \\
43049 \mathrm{i}\end{array}$ & $2.18 \mathrm{~b}$ & 7.44c & 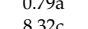 & $\begin{array}{l}7.56 c \\
5701 \mathrm{e}\end{array}$ & $\begin{array}{l}9.15 \mathrm{~d} \\
5191 \mathrm{e}\end{array}$ & $30.92 \mathrm{f}$ & $0.28 \mathrm{a}$ \\
\hline $\begin{array}{l}\mathrm{A} 8 \\
\mathrm{~A} 9\end{array}$ & $\begin{array}{l}2.35 \mathrm{~b} \\
0.31 \mathrm{a}\end{array}$ & $\begin{array}{l}0.09 \mathrm{a} \\
0.03 \mathrm{a}\end{array}$ & $\begin{array}{l}1.41 \mathrm{a} \\
0.06 \mathrm{a}\end{array}$ & $\begin{array}{l}8.25 \mathrm{c} \\
1.85 \mathrm{~b}\end{array}$ & $\begin{array}{l}\begin{array}{r}255.7 .7 \mathrm{~g} \\
5.68 \mathrm{c}\end{array} \\
\end{array}$ & $\begin{array}{l}13.27 \mathrm{da} \\
0.27 \mathrm{a}\end{array}$ & $\begin{array}{l}8.3 .55 \mathrm{f} \\
62.84 \mathrm{~g}\end{array}$ & $\begin{array}{l}83.05 \mathrm{f} \\
6.05 \mathrm{c}\end{array}$ & 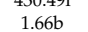 & $\begin{array}{c}-\bar{c} \\
4.30 \mathrm{c}\end{array}$ & $\begin{array}{l}32.1 / 2 \mathrm{n} \\
22.36 \mathrm{f}\end{array}$ & $\begin{array}{l}8.32 \mathrm{C} \\
0.80 \mathrm{a}\end{array}$ & $\begin{array}{l}5 / .01 \mathrm{e} \\
5.07 \mathrm{c}\end{array}$ & $\begin{array}{l}\text { S1.91e } \\
8.64 \mathrm{de}\end{array}$ & $\begin{array}{l}27.200 \mathrm{~g} \\
12.12 \mathrm{e}\end{array}$ & $\begin{array}{l}.131 c \\
0.39 \mathrm{a}\end{array}$ \\
\hline A10 & $0.07 a$ & - & $0.04 a$ & $1.13 \mathrm{ab}$ & $8.10 \mathrm{c}$ & $0.45 a$ & $36.74 \mathrm{e}$ & $4.97 \mathrm{~b}$ & $2.84 \mathrm{ab}$ & $2.26 \mathrm{ab}$ & $14.58 \mathrm{~d}$ & $1.42 \mathrm{ab}$ & $5.73 \mathrm{~b}$ & $8.70 \mathrm{c}$ & $15.52 \mathrm{~d}$ & $0.41 a$ \\
\hline Total alkanes & $3.77 \mathrm{~b}$ & $0.56 \mathrm{a}$ & $2.13 \mathrm{~b}$ & $22.54 \mathrm{~d}$ & $355.24 \mathrm{~h}$ & 23.51d & $343.27 \mathrm{~h}$ & $108.66 \mathrm{f}$ & $583.37 \mathrm{j}$ & $11.07 \mathrm{c}$ & $452.51 \mathrm{i}$ & $16.98 \mathrm{c}$ & 83.74e & $156.36 \mathrm{~g}$ & $721.18 \mathrm{k}$ & $13.24 \mathrm{c}$ \\
\hline Others & & & & & & & & & & & & & & & & \\
\hline $\mathrm{O} 1$ & - & - & - & - & - & $1.29 \mathrm{a}$ & - & - & - & - & - & - & 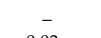 & - & - & 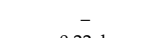 \\
\hline $\mathrm{O} 2$ & - & 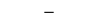 & - & - & 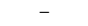 & - & tr & $0.33 b$ & - & - & $0.62 \mathrm{c}$ & - & $0.03 a$ & tr & - & $0.22 \mathrm{ab}$ \\
\hline O4 & - & - & - & - & $0.83 \mathrm{~b}$ & - & - & - & - & - & - & $\operatorname{tr}$ & - & - & $4.12 \mathrm{c}$ & $0.07 \mathrm{a}$ \\
\hline O5 & - & $0.07 a$ & - & - & $80.61 \mathrm{~b}$ & - & - & -- & - & - & - & - & - & - & 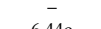 & - \\
\hline $\begin{array}{l}\text { O6 } \\
\text { Total volatiles }\end{array}$ & $10-93 \mathrm{~b}$ & $\begin{array}{c}- \\
5.85 \mathrm{a}\end{array}$ & $10-85 \mathrm{~b}$ & $\begin{array}{r}1.54 b \\
145.69 \mathrm{e}\end{array}$ & $\begin{array}{c}4.18 \mathrm{~d} \\
1018.79 \mathrm{i}\end{array}$ & $\begin{array}{l}0.43 \mathrm{a} \\
68.27 \mathrm{~d}\end{array}$ & $\begin{array}{l}7.46 \mathrm{e} \\
782.98 \mathrm{~h}\end{array}$ & $\begin{array}{l}2.51 \mathrm{c} \\
652.56 \mathrm{~h}\end{array}$ & $\begin{array}{l}19.84 \mathrm{~g} \\
1293.25 \mathrm{j}\end{array}$ & $\begin{array}{l}\text { 1.1.10ab } \\
223.22 \mathrm{f}\end{array}$ & $\begin{array}{c}14.591 \mathrm{f} \\
1999.09 \mathrm{k}\end{array}$ & $\begin{array}{l}1.33 b \\
30.53 c\end{array}$ & $\begin{array}{l}5.32 \mathrm{de} \\
421.5 \mathrm{~g}\end{array}$ & $\begin{array}{l}6.76 \mathrm{e} \\
972.33 \mathrm{i}\end{array}$ & $\begin{array}{l}6.44 \mathrm{e} \\
2327.611\end{array}$ & $\begin{array}{l}0.84 \mathrm{a} \\
51.52 \mathrm{~d}\end{array}$ \\
\hline
\end{tabular}

${ }^{1}$ Means with different letters in the same row for each volatile indicate significant differences at $p<0.05$ according to Newman-Keuls test. ${ }^{2}$ tr indicates traces $\left(<0.01 \times 10^{6}\right.$ GC peak area units). 
Generally, the most common and abundant terpenoids in placental tissues were again 3-carene, $\alpha$-copaene, $\alpha$-muurolene, valencene, and $\beta$-himachalene. Contents of $\alpha$-longipinene, $\alpha$-himachalene, and longipinocarvone appeared in many accessions but at low levels. Finally, $\delta$-cadinene and $\beta$-elemene reached remarkably high levels in 'Aji Dulce' and 'Cayenne', respectively, while the rest of the terpenoids were found at trace or low amounts (Table 4). The most abundant compound was valencene in 'Chile Serrano', 'Cayenne', PI-152225, and 'Chile de Arbol', while $\alpha$-muurolene was the most abundant compound in 'Serrano Criollo' (Table 4). As observed in the fruit pericarps, many accessions had one predominant terpenoid compound. Valencene contributed $80-95 \%$ of the total terpenoids in 'Chile de Arbol', 'Cayenne', 'Chile Serrano', and PI-152225, and the same was found for 3-carene in blocky peppers and $\alpha$-murolene in 'Serrano Criollo'. In contrast, 'Aji Dulce' showed a more balanced distribution (Table 4).

The qualitative and quantitative contributions of esters were considerably lower than the terpenoids. Nonetheless, their abundance was comparatively higher in placental tissues than the pericarp. Again, C. chinense PI-152225 had an abundant and diverse profile, but other C. annuum accessions, like 'Cayenne' and 'Serrano Criollo' also had several esters in their composition and at remarkably high levels (Table 4). Finally, 'Pasilla Bajio', 'Chile Serrano', and 'Aji Dulce' also had some esters, although only the latter reached high levels (Table 4). Hexyl 2,2-dimethylpropanoate was the most common and abundant ester, followed by high levels of pentyl 4-methylpentanoate and 4-hexen-1-yl-pentanoate. These findings indicated that the placental tissues of peppers may have more intense fruity notes than those found in their pericarps, and may explain why sauces and pastes formulated with seeds and placenta have hotter and more attractive flavors $[4,5,13,14]$.

The profiles of alkanes in most parents were complex in the placental tissues, and particularly in the hot peppers: 'Chile de Arbol', 'Cayenne', 'Pasilla Bajio', 'Chile Serrano', and PI-152225 (Table 4). Similar to the pericarp, eicosane, nonadecane, tetradecane, 10-methylnonadecane, and 8-hexylpentadecane were the most common alkanes, and cyclopentadecane was also abundant in PI-152225 and 'Chile Serrano'. Finally, among the rest of volatiles, (Z)-9-octadecen-1-ol was present in all the accessions with the only exception of Blocky and 'Piquillo' peppers, while the rest of the miscellaneous compounds were very rare, and only (E)-2-hexadecen-1-ol was present at high levels in 'Cayenne'. Furthermore, the bell pepper pyrazine only appeared in the placenta of Serrano peppers and at very low levels, and even methyl salicylate was absent in this part of the fruit, in contrast to the results obtained for the pericarp (Table 4), which suggests that this part of the fruit may have lower or no green paprika sulfurous notes in most genotypes.

\subsubsection{Hybrid Accessions}

The volatile composition in the placental tissues of the hybrids showed similarities but also differences within the pericarp. The qualitative increase due to hybridization was generally lower in the placental tissues in comparison to the pericarp. Thus, the number of volatiles detected in the hybrids ranged from 18 to 23, while the parents showed a similar range (10 to 24) and many parents showed $\geq 18$ volatiles (Table 4). Furthermore, half of the hybrids (Piquillo $\times$ Pasilla, Piquillo $\times$ Serrano and CayennexSerrano) showed a number of volatiles comprised between those of the corresponding parent accessions, although close to the parent with the highest number (Table 4). Considering the total volatiles, again, the two different inheritance models were found in the same hybrids: (i) hybrids with intermediate levels in comparison to their parent lines. i.e., Piquillo $\times$ Pasilla, Piquillo $\times$ Serrano, and BlockyR1×PI152225, and (ii) transgressive hybrids, like Chile de Arbol $\times$ Serrano, and others like BlockyR1 $\times$ Serrano Criollo and CayenneXSerrano, which showed higher amounts of total volatiles (2-3-fold) than the parent with the highest levels (Table 4). Therefore, our results indicated that hybridization may provide a remarkable increase in the total volatiles and the complexity of the volatile fraction in both parts of the fruits, although the increase in complexity may be lower in placental tissues.

Regarding the inheritance of individual volatiles, different cases of intermediate or transgressive inheritance were detected and the mode of inheritance differed greatly among hybrids. In addition, 
many cases of new volatiles due to genetic complementation were also found (Table 4). Thus, a balanced distribution of transgressive and intermediate inheritance cases was predominant in hybrids like PiquilloxPasilla, Arbol $\times$ Serrano, and Cayenne $\times$ Serrano, while others like Piquillo $\times$ Serrano and BlockyR1XPI152225 showed intermediate inheritance in most volatiles combined with some cases of de novo volatiles (Table 4). Finally, in BlockyR1×Serrano Criollo, the cases of de novo volatiles or disappearance of other volatiles were predominant (Table 4).

\subsection{Comparative Accumulation of Volatiles in Fruit Tissues}

Apart from a few exceptions, the comparison of the volatile fractions from both fruit tissues revealed two main facts: (i) within each accession, the volatile fraction in the placenta showed higher levels than in the pericarp in both total volatiles and most individual compounds, and (ii) the higher abundance of volatiles in the pericarp, the higher in the placental tissue as well.

In this way, those parent accessions with the most abundant volatile fractions in the pericarp, i.e., 'Cayenne', 'Chile Serrano', and PI152225, also showed a remarkably high content and diverse profile of volatiles in the placental tissues, while the contrary was true in Blocky and 'Piquillo' peppers, which showed the lowest content in volatiles and least diverse volatile compositions in both tissues (Tables 3 and 4). In addition, a similar trend was found in the hybrids as those with the most diverse and abundant volatiles in the placental tissues also showed high levels and very diverse profiles in pericarp composition, e.g., CayenneXSerrano, Arbol $\times$ Serrano (Tables 3 and 4).

Furthermore, this tissue effect was remarkable within each parent accession and hybrid. For instance, most accessions showed placenta/pericarp ratios $>4$ in the total volatiles, like BlockyR1 and 'Aji Dulce' (8-fold and 4-fold, respectively), 'Serrano Criollo' (24-fold), or hybrids like BlockyR1×SerrCrio (27-fold) and BlockyR1×152225 (40-fold) (Tables 3 and 4). These results suggest that volatile accumulation was more pronounced in the inner parts of the fruits, close to the seed area. A similar gradient of fruit composition has been described in many volatiles and other metabolites in peach, melon, tomato, or passion fruit, among other fruit species [45-48] and suggests a more intense metabolism of volatiles in this part of the peppers. In this regard, the phenylpropanoid pathway plays an important role in the remarkably high accumulation of capsaicinoids in the placenta of peppers against mammal herbivores. Similarly, higher levels of other compounds, like for instance those related to flavor and aroma, might be also expected in these tissues as a consequence of up-regulated metabolism $[13,29,49,50]$. In addition, such richness of volatiles in the placental tissues should be considered by food processors and breeders aiming to achieve and formulate sauces and pastes with more intense flavor and pungency (as capsaicinoids also accumulate mainly in these tissues).

The hierarchical cluster analyses illustrated these differences and similarities in volatile fractions. Considering the parental accessions, the analysis generated two main clusters: (i) cluster A, including 'Serrano Criollo' (subcluster A1) and 'Chile de Arbol', 'Chile Serrano', and the placental fraction of 'Cayenne' (subcluster A2), and (ii) cluster B, which included the rest of the evaluated parent accessions and tissues, with PI152225 appearing separately in subcluster B1 (Figure 1).

The pericarp and placental profiles of each accession clustered together (i.e., 'Pasilla Bajio', BlockyR2, 'Aji Dulce', PI-152225, 'Chile de Arbol', 'Chile Serrano', and 'Serrano Criollo'), confirming the strong genotypic factor mentioned above (Figure 1). The only exceptions where the pericarp and placental samples from each accession appeared separately were found in BlockyR1, 'Piquillo', and 'Cayenne'. In the case of BlockyR1 and 'Piquillo', this was mainly due to their low content and diversity of volatiles, which increased their similarity. Only 'Cayenne' clearly displayed its pericarp and placental profiles in separate clusters, which means that exceptionally great differences can be found between fruit tissues in certain genotypes.

Similarly to the parent accessions, the pericarp and placental profiles of the hybrids were grouped in two main clusters, and the profiles of both tissues within each hybrid were clustered in pairs in most cases, i.e., Cayenne $\times$ Serrano, BlockyR1 $\times$ SerrCrio, BlockyR1 $\times$ PI-152225, and Piquillo $\times$ Pasilla, due to the similarity between the volatile composition of their fruit tissues. By contrast, Piquillo $\times$ Serrano and 
Arbol $\times$ Serrano displayed their pericarp and placental samples in different clusters of the heatmap (Figure 2). Furthermore, pericarp samples from BlockyR1×AjiD and BlockyR2 $\times$ AjiD clustered together.

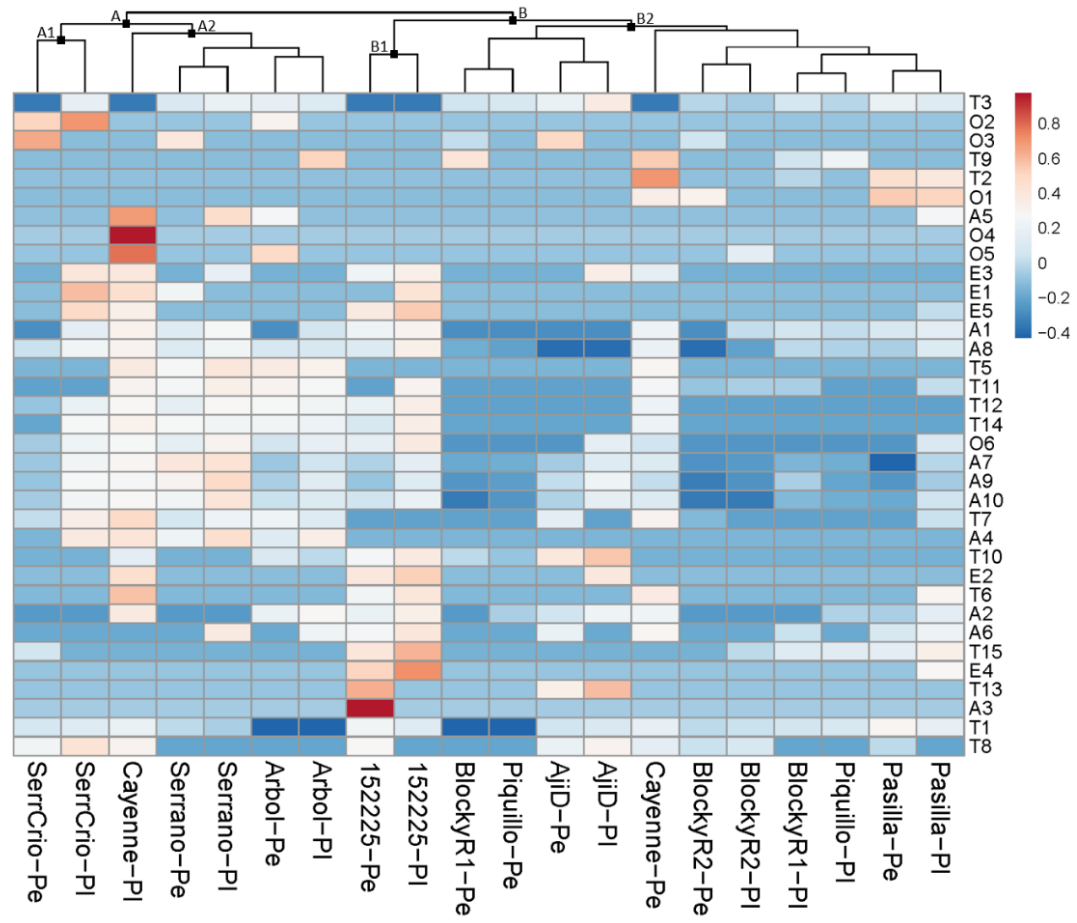

Figure 1. Heatmap hierarchical cluster analysis of the volatiles identified in the parent accessions studied, corresponding to the pericarp (Pe) and placental (Pl) tissues. Accession and volatile abbreviations correspond to those indicated in Tables 1 and 2, respectively.

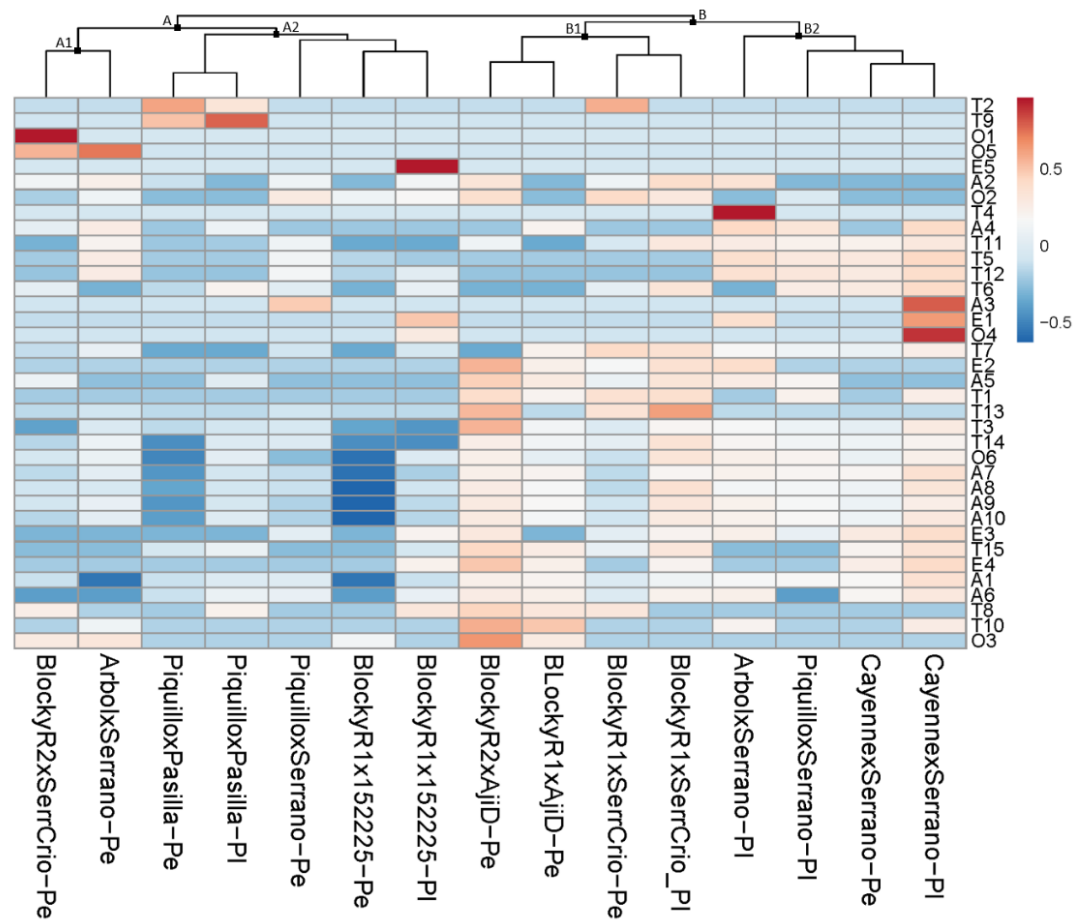

Figure 2. Heatmap hierarchical cluster analysis of the volatiles identified in the hybrid accessions studied, corresponding to the pericarp (Pe) and placental (Pl) tissues. Accession and volatile abbreviations correspond to those indicated in Tables 1 and 2, respectively. 
According to the principal component analysis, the two main components explained $43 \%$ of the total variation, and PC1 contributed to about $30 \%$ of the variation (Figure 3). PC1 was mainly related positively to several terpenoids, including the highly abundant 3-carene (T1), cyclosativene (T2), and $\beta$-selinene (T9), and the heterocyclic compound indole (O1); and negatively related to many other compounds, including several alkanes, like tetradecane (A1), cyclopentadecane (A6), and 10-methylnonadecane (A8); terpenoids, like $\beta$-elemene (T6), valencene (T11), $\alpha$-himachalene (T12), $\beta$-himachalene (T14), and longipinocarvone (T15); and most esters and other compounds, like $\alpha$-ionone (O4) and (Z)-9-octadecen-1-ol (O6) (Figure 3, top). Regarding PC2, the most discriminant volatiles in the positive side of PC2 were the terpenoids allo-aromadendrene (T5), $\beta$-elemene (T6), and $\alpha$-himachalene (T12), the alkanes 4-methyltetradecane (A3), decylcyclopentane (A4), and $\alpha$-ionone (O4), while the terpenoids $\alpha$-copaene (T3), longipinocarvone (T15), and the miscellaneous compounds methyl salicylate (O3) and 3-isobutyl-2-methoxypyrazine (O2) were found in the negative extreme (Figure 3).
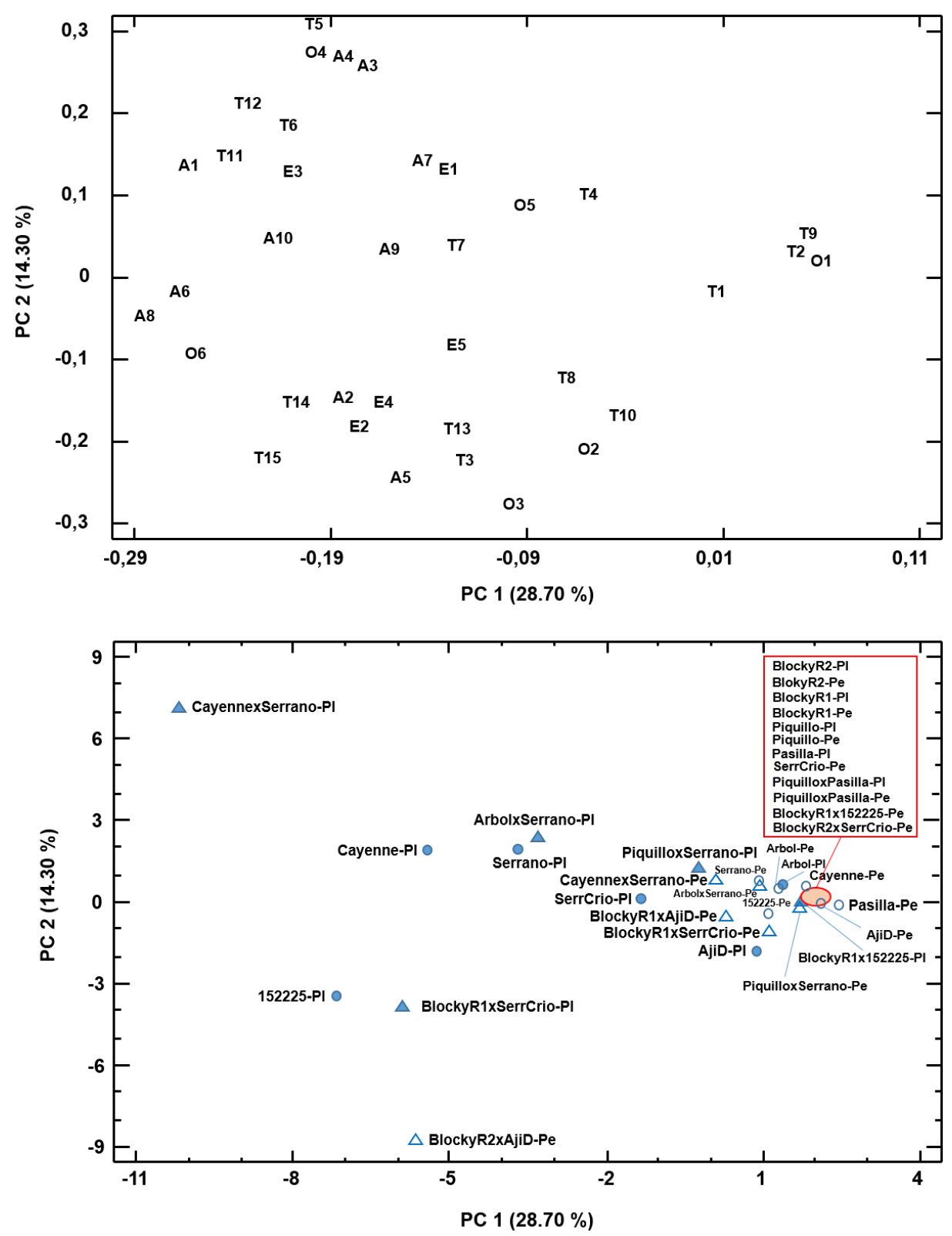

Figure 3. Principal component analysis (PCA) plots of the volatiles identified (upper) and the parent and hybrid accessions evaluated, corresponding to the pericarp (Pe) and placental (Pl) tissues (bottom). Accession and volatile abbreviations correspond to those indicated in Tables 1 and 2, respectively. 
In agreement with the PCA distribution observed for the volatiles, both pericarp and placenta samples from all parent accessions grouped in subcluster B2 in Figure 1 (apart from the placenta of 'Aji Dulce') and all hybrids grouped in cluster A in Figure 2 (apart from the pericarp of Arbol $\times$ Serrano) concentrated in a core group (Figure 3 bottom, red circle) in the positive side of $\mathrm{PC} 1$ and the intermediate level (i.e., 0 value) of PC2. The pericarp fractions of 'Chile de Arbol', 'Chile Serrano', and PI-152225, and the hybrids Arbol $\times$ Serrano, Cayenne $\times$ Serrano, BlockyR1 $\times$ AjiD, and BlockyR1 $\times$ SerrCrio, as well as the placenta fractions of 'Chile de Arbol' and 'Aji Dulce', were close to this core group (Table 3).

In general, most of these samples were characterized by low or intermediate amounts of total volatiles and low to intermediate complexity in the composition of the volatile fraction, where some terpenoids, like 3-carene, were also predominant and esters and other additional compounds (group $\mathrm{O}$ ) were very scarce or absent. As a result, most of the samples with green paprika aroma and very little or no fruity notes were located in this region.

By contrast, the placental fractions of the rest of the accessions were located out of the mentioned cloud. In this regard, the 'Serrano Criollo' and PiquilloxSerrano placental samples, characterized by their intermediate amounts of total volatiles and complexity of volatile profiles, were the closest ones to the mentioned cloud (Figure 3). Advancing gradually towards the negative values of PC1, a second group including the placenta fractions of 'Chile Serrano', 'Cayenne', and the Arbol×Serrano hybrid, all of them characterized by high amounts of volatiles and complex volatile profiles (including mixtures of terpenoids with some esters and miscellaneous compounds), and therefore with intermediate-highly intense green paprika aromas mixed with fruity notes, appeared clearly separated from the central cloud (Figure 3).

Finally, the rest of the studied volatile fractions were found at the negative side of PC1, due to their diverse and abundant composition in esters (related to fruity aromas), alkanes (tentatively related to capsaicinoids), and several terpenoids, like valencene. These samples were distributed along the PC2 according to their differences in terpenoids, esters, and some miscellaneous compounds (Figure 3). Furthermore, the placenta fraction from Cayenne $\times$ Serrano, which showed the highest total volatiles of the experiment and one of the most complex profiles (Tables 3 and 4), were clearly separated from the rest of samples, with the most negative PC1 values and the most positive PC2 values (Figure 3).

In view of these findings, hybridization allowed more complex and usually more abundant volatile fractions than those observed in the original parents, widening and improving the richness and complexity of volatiles. As a result, many hybrids spread widely in the PCA plot and many of them separately from their parents. This is particularly obvious in the case of placental tissues, which, due to their usually higher levels in volatiles, showed a wider distribution in the PCA (Figure 3).

\section{Conclusions}

In conclusion, this work demonstrated that there are ample opportunities to improve the aroma-related compounds in fully ripe Capsicum peppers by hybridization, both qualitatively and quantitatively. Most hybrids showed more complex volatile profiles than those of their corresponding parents as a result of the confluence of volatiles from both parent lines, with cases of intermediate inheritance or transgressive inheritance, and also, but to a lesser extent, to the appearance of new compounds, presumably due to genetic complementation. Thus, breeders can plan sets of hybrid combinations using parents with complementary volatile fractions, and then perform final selections of those hybrids with the most desirable flavors and aromas based on organoleptic tests. The two main fruit parts-the pericarp and placental tissues-followed these inheritance rules. In general, placental tissues were quantitatively richer in volatile compounds, which is of special interest for breeding the aroma and flavor of varieties where the whole fruit is used in culinary applications.

Author Contributions: E.M.-P., A.F. and A.R.-B. conceived and designed the experiment; E.M.-P., M.C.G.-M. and C.C.-O. conducted the experiment, collected the data and performed the analyses; E.M.-P., M.D.-D. and A.d.L.-M. performed data processing and statistical analyses; A.F., C.C.-O. and A.R.-B. provided the facilities; E.M.-P. and C.C.-O. wrote the draft manuscript; M.D.-D, A.d.L.-M., M.C.G.-M., A.F. and A.R.-B. provided advice 
and revisions during the preparation of the manuscript; E.-M.P. and C.-C.O. edited the manuscript; M.-D.D. and A.-R.B. supervised the whole preparation of manuscript A.-R.B. was in charge of Project administration and funding acquisition. All authors have read and agree to the published version of the manuscript.

Funding: This work has been funded by INIA project RTA2014-00041-C02-02, FEDER Funds.

Acknowledgments: Authors thank Jaime Prohens for his advice in statistics and Javier Díez Duet for his support with the edition of the manuscript.

Conflicts of Interest: The authors declare no competing financial interests.

\section{References}

1. Garcés-Claver, A.; Arnedo-Andrés, M.S.; Abadía, J.; Gil-Ortega, R.; Álvarez-Fernández, A. Determination of capsaicin and dihydrocapsaicin in Capsicum fruits by liquid chromatography-electrospray/time-of-flight mass spectrometry. J. Agric. Food Chem. 2006, 54, 9303-9311. [CrossRef] [PubMed]

2. López-Castilla, L.C.; Garruña-Hernández, R.; Castillo-Aguilar, C.C.; Martínez-Hernández, A.; Ortíz-García, M.M.; Andueza-Noh, R.H. Structure and Genetic Diversity of Nine Important Landraces of Capsicum Species Cultivated in the Yucatan Peninsula, Mexico. Agronomy 2019, 9, 376. [CrossRef]

3. Pereira-Dias, L.; Vilanova, S.; Fita, A.; Prohens, J.; Rodríguez-Burruezo, A. Genetic diversity, population structure, and relationships in a collection of pepper (Capsicum spp.) landraces from the Spanish centre of diversity revealed by genotyping-by-sequencing (GBS). Hortic. Res. 2019, 6, 54. [CrossRef] [PubMed]

4. Patel, K.; Ruiz, C.; Calderon, R.; Marcelo, M.; Rojas, R. Characterisation of volatile profiles in 50 native Peruvian chili pepper using solid phase microextraction-gas chromatography mass spectrometry (SPME-GCMS). Food Res. Int. 2016, 89, 471-475. [CrossRef] [PubMed]

5. DeWitt, D.; Bosland, P.W. The Complete Chile Pepper Book; Timber Press: Portland, OR, USA, 2009.

6. Bosland, P.W.; Votava, E. Peppers: Vegetable and Spice Capsicums. CABI Publishing: New York, NY, USA, 2000.

7. Ribes-Moya, A.M.; Raigón, M.D.; Moreno-Peris, E.; Fita, A.; Rodríguez-Burruezo, A. Response to organic cultivation of heirloom Capsicum peppers: Variation in the level of bioactive compounds and effect of ripening. PLoS ONE 2018, 13, e0207888. [CrossRef]

8. Pino, J.; González, M.; Ceballos, L.; Centurión-Yah, A.R.; Trujillo-Aguirre, J.; Latourniere-Moreno, L.; Sauri-Duch, E. Characterization of total capsaicinoids, colour and volatile compounds of Habanero chilli pepper (Capsicum chinense Jack.) cultivars grown in Yucatan. Food Chem. 2007, 104, 1682-1686. [CrossRef]

9. Rodríguez-Burruezo, A.; Kollmannsberger, H.; González-Mas, M.C.; Nitz, S.; Nuez, F. HS-SPME comparative analysis of genotypic diversity in volatile fraction and aroma contributing compounds of Capsicum fruits from the annuum-chinense-frutescens complex. J. Agric. Food Chem. 2010, 58, 4388-4400. [CrossRef]

10. Bogusz, S., Jr.; Teixeira-Godoy, H.; Marchi-Tavares, A.; Teixeira, J. Analysis of the volatile compounds of Brazilian chilli peppers (Capsicum spp.) at two stages of maturity by solid phase micro-extraction and gas chromatography-mass spectrometry. Food Res. Int. 2012, 48, 98-107. [CrossRef]

11. Morales-Soriano, E.; Kebede, B.T.; Ugás, R.; Grauwet, T.; Van Loey, A.; Hendrickx, M. Flavor characterization of native Peruvian chili peppers through integrated aroma fingerprinting and pungency profiling. Food Res. Int. 2018, 109, 250-259. [CrossRef]

12. Buczkowska, H.; Nurzynska-Wierdak, R.; Labuda, H.; Salata, A. Relationships of the capsaicinoid content between the fruit parts of hot pepper (Capsicum annuum L.). Acta Sci. Pol. Hortorum Cultus. 2016, 15, 185-198.

13. Olguín-Rojas, J.A.; Fayos, O.; Vázquez-León, L.A.; Ferreiro-González, M.; Rodríguez-Jimenes, G.C.; Palma, M.; Garcés-Claver, A.; Barbero, G.F. Progression of the Total and Individual Capsaicinoids Content in the Fruits of Three Different Cultivars of Capsicum chinense Jacq. Agronomy 2019, 9, 141. [CrossRef]

14. Baby, K.C.; Ranganathan, T.V. Effect of enzyme pretreatment on yield and quality of fresh green chilli (Capsicum annuum L.) oleoresin and its major capsaicinoids. Biocatal. Agric. Biotechnol. 2016, 7, 95-101. [CrossRef]

15. Barchenger, D.W.; Bosland, P.W. Exogenous applications of capsaicin inhibits seed germination of Capsicum Annuum. Sci. Hortic. 2016, 203, 29-31. [CrossRef]

16. Scoville, W.L. Note on Capsicum. J. Am. Pharm. Assoc. 1912, 1, 453-454. [CrossRef]

17. Estrada, B.; Pomar, F.; Díaz, J.; Merino, F.; Bernal, M.A. Pungency level in fruits of the Padrón pepper with different water supply. Sci. Hort. 1999, 81, 385-396. [CrossRef] 
18. Jarret, R.L.; Perkins, B.; Fan, T.; Prince, A.; Guthrie, K.; Skoczenski, B. Using EIA to screen Capsicum spp. Germplasm for capsaicinoid content. J. Food Comp. Anal. 2003, 16, 189-194. [CrossRef]

19. Wahyuni, Y.; Ballester, A.R.; Sudarmonowati, E.; Bino, R.J.; Bovy, A.G. Secondary metabolites of Capsicum species and their importance in the human diet. J. Nat. Prod. 2013, 76, 783-793. [CrossRef]

20. Lu, M.; Ho, C.T.; Huang, Q. Extraction, bioavailability, and bioefficacy of capsaicinoids. J. Food Drug Anal. 2017, 25, 27-36. [CrossRef]

21. Kollmannsberger, H.; Rodríguez-Burruezo, A.; Nitz, S.; Nuez, F. Volatile and capsaicinoid composition of ají (Capsicum baccatum) and rocoto (Capsicum pubescens), two Andean species of chile peppers. J. Sci. Food Agric. 2011, 91, 1598-1611. [CrossRef]

22. Eggink, P.M.; Tikunov, Y.; Maliepaard, C.; Haanstra, J.P.; de Rooij, H.; Vogelaar, A.; Gutteling, E.W.; Freymark, G.; Bovy, A.G.; Visser, R.G. Capturing flavors from Capsicum baccatum by introgression in sweet pepper. Theor. Appl. Genet. 2014, 127, 373-390. [CrossRef]

23. Luning, P.A.; de Rijk, T.; Wichers, H.J.; Roozen, J.P. Gas chromatography, mass spectrometry, and sniffing port analyses of volatile compounds of fresh bell peppers (Capsicum annuum) at different ripening stages. J. Agric. Food Chem. 1994, 42, 977-983. [CrossRef]

24. Cremer, D.R.; Eichner, K. Formation of volatile compounds during heating of spice paprika (Capsicum annuum) powder. J. Agric. Food Chem. 2000, 48, 2454-2460. [CrossRef] [PubMed]

25. Hammer, K.; Arrowsmith, N.; Gladis, T. Agrobiodiversity with emphasis on plant genetic resources. Naturwissenschaften 2003, 90, 241-250. [CrossRef] [PubMed]

26. Brugarolas, M.; Martinez-Carrasco, L.; Martinez-Poveda, A.; Ruiz, J.J. A competitive strategy for vegetable products: Traditional varieties of tomato in the local market. Span. J. Agric. Res. 2009, 7, 294-304. [CrossRef]

27. Gancel, A.L.; Ollitrault, P.; Froelicher, Y.; Tomi, F.; Jacquemond, C.; Luro, F.; Brillouet, J.M. Leaf volatile compounds of six citrus somatic allotetraploid hybrids originating from various combinations of lime, lemon, citron, sweet orange, and grapefruit. J. Agric. Food Chem. 2005, 53, 2224-2230. [CrossRef]

28. Rodríguez-Burruezo, A.; Kollmannsberger, H.; Prohens, J.; Nitz, S.; Nuez, F. Analysis of the volatile aroma constituents of parental and hybrid clones of pepino (Solanum muricatum). J. Agric. Food Chem. 2004, 52, 5663-5669. [CrossRef]

29. Antonio, A.S.; Wiedemann, L.S.M.; Veiga-Junior, V.F. The genus Capsicum: A phytochemical review of bioactive secondary metabolites. RSC Adv. 2018, 8, 25767-25784. [CrossRef]

30. Zimmermann, M.; Schieberle, P. Important odorants of sweet bell pepper powder (Capsicum annuum cv annuиm): Differences between samples of Hungarian and Morrocan origin. Eur. Food Res. Technol. 2000, 211, 175-180. [CrossRef]

31. Simian, H.; Robert, F.; Blank, I. Identification and synthesis of 2-heptanethiol, a new flavor compound found in bell peppers. J. Agric. Food Chem. 2004, 52, 306-310. [CrossRef]

32. Sosa-Moguel, O.; Pino, J.A.; Ayora-Talavera, G.; Sauri-Duch, E.; Cuevas-Glory, L. Biological activities of volatile extracts from two varieties of Habanero pepper (Capsicum chinense Jacq.). Int. J. Food Prop. 2017, 20 (Suppl. 3), S3042-S3051. [CrossRef]

33. Bianchi, G.; Lo Scalzo, R. Characterization of hot pepper spice phytochemicals, taste compounds content and volatile profiles in relation to the drying temperature. J. Food Biochem. 2018, 42, e12675. [CrossRef]

34. Moreno, E.; Fita, A.; González-Mas, M.C.; Rodríguez-Burruezo, A. HS-SPME study of the volatile fraction of Capsicum accessions and hybrids in different parts of the fruit. Sci. Hort. 2012, 135, 87-97. [CrossRef]

35. Gómez, E.; Ledbetter, C.A.; Hartsell, P.L. Volatile compounds in apricot, plum, and their interspecific hybrids. J. Agric. Food Chem. 1993, 41, 1669-1676. [CrossRef]

36. Gancel, A.L.; Ollitrault, P.; Froelicher, Y.; Tomi, F.; Jacquemond, C.; Luro, F.; Brillouet, J.M. Leaf volatile compounds of seven citrus somatic tetraploid hybrids sharing willow leaf mandarin (Citrus deliciosa Ten.) as their common parent. J. Agric. Food Chem. 2003, 51, 6006-6013. [CrossRef]

37. Nuez, F.; Gil, R.; Costa, J. El Cultivo de Pimientos, Chiles y Ajíes (The Crop of Peppers, Chilies and Ajíes); MundiPrensa: Madrid, Spain, 2003. (In Spanish)

38. Metsalu, T.; Vilo, J. ClustVis: A web tool for visualizing clustering of multivariate data using principal component analysis and heatmap. Nucleic Acids Res. 2015, 43, W566-W570. [CrossRef]

39. Cuevas-Glory, L.F.; Sosa-Moguel, O.; Pino, J.; Sauri-Duch, E. GC-MS characterization of volatile compounds in habanero pepper (Capsicum chinense Jacq.) by optimization of headspace solid-phase microextraction conditions. Food Anal. Method. 2015, 8, 1005-1013. [CrossRef] 
40. Orzáez, D.; Granell, A. Reverse genetics and transient gene expression in fleshy fruits: Overcoming plant stable transformation. Plant Signal. Behav. 2009, 4, 864-867. [CrossRef]

41. Pinheiro, T.T.; Peres, L.E.P.; Purgatto, E.; Latado, R.R.; Maniero, R.A.; Martins, M.M.; Figueira, A. Citrus carotenoid isomerase gene characterization by complementation of the "Micro-Tom" tangerine mutant. Plant Cell Rep. 2019, 38, 623-636. [CrossRef]

42. Rothan, C.; Diouf, I.; Causse, M. Trait discovery and editing in tomato. Plant J. 2019, 97, 73-90. [CrossRef] [PubMed]

43. Goulet, B.E.; Roda, F.; Hopkins, R. Hybridization in plants: Old ideas, new techniques. Plant Physiol. 2017, 173, 65-78. [CrossRef] [PubMed]

44. Rambla, J.L.; Tikunov, Y.M.; Monforte, A.J.; Bovy, A.G.; Granell, A. The expanded tomato fruit volatile landscape. J. Exp. Bot. 2014, 65, 4613-4623. [CrossRef] [PubMed]

45. Aubert, C.; Milhet, C. Distribution of the volatile compounds in the different parts of a white-fleshed peach (Prunus persica L. Batsch). Food Chem. 2007, 102, 375-384. [CrossRef]

46. Moing, A.; Aharoni, A.; Biais, B.; Rogachev, I.; Meir, S.; Brodsky, L.; Allwood, J.W.; Erban, A.; Dunn, W.B.; Kay, L.; et al. Extensive metabolic cross-talk in melon fruit revealed by spatial and developmental combinatorial metabolomics. New Phytol. 2011, 190, 683-696. [CrossRef]

47. Deyeye, E.; Aremu, M. Chemical composition of theraw fruit coat, seed and pulp of passion fruit (Passiflora edulis). J. Trends Sci. Tech. 2017, 2, 334-341.

48. Wang, L.; Qian, C.; Bai, J.; Luo, W.; Jin, C.; Yu, Z. Difference in volatile composition between the pericarp tissue and inner tissue of tomato (Solanum lycopersicum) fruit. J. Food Process. Preserv. 2018, 42, e13387. [CrossRef]

49. Dardick, C.; Callahan, A.M. Evolution of the fruit endocarp: Molecular mechanisms underlying adaptations in seed protection and dispersal strategies. Front Plant Sci. 2014, 5, 284. [CrossRef]

50. Bosland, P.; Coon, D.; Cooke, P.H. Novel formation of ectopic (nonplacental capsaicinoid secreting vesicles on fruit walls explains the morphological mechanism for super-hot chile peppers. J. Amer. Soc. Hort. Sci. 2015, 140, 253-256. [CrossRef] 